\title{
Article \\ A Theoretical Model to Identify the Fundamental Frequency of Simply Supported Girders from a Passing Heavy Vehicle
}

\author{
Qi Wu ${ }^{1}$, Bing Han ${ }^{1,2}, * \mathbb{D}$, Jiaping Yu ${ }^{1}$, Wutong Yan ${ }^{1}$, Jinquan Zhang ${ }^{3}$ and Huibing Xie ${ }^{1,2} \mathbb{D}$ \\ 1 School of Civil Engineering, Beijing Jiaotong University, No.3 Shangyuancun, Haidian District, \\ Beijing 100044, China; 16115271@bjtu.edu.cn (Q.W.); jiapingyu@bjtu.edu.cn (J.Y.); \\ 15115261@bjtu.edu.cn (W.Y.); hbxie@bjtu.edu.cn (H.X.) \\ 2 Key Laboratory of Safety and Risk Management on Transport Infrastructures, Ministry of Transport of the \\ People's Republic of China, Beijing 100044, China \\ 3 Research Institute of Highway Ministry of Transportation, West Tucheng Road 8, Haidian District, \\ Beijing 100088, China; jq.zhang@rioh.cn \\ * Correspondence: bhan@bjtu.edu.cn
}

check for updates

Citation: Wu, Q.; Han, B.; Yu, J.; Yan, W.; Zhang, J.; Xie, H. A Theoretical Model to Identify the Fundamental Frequency of Simply Supported Girders from a Passing Heavy Vehicle. Appl. Sci. 2022, 12, 2422. https://doi.org/10.3390/ app12052422

Academic Editor: César M. A. Vasques

Received: 10 January 2022

Accepted: 19 February 2022

Published: 25 February 2022

Publisher's Note: MDPI stays neutral with regard to jurisdictional claims in published maps and institutional affiliations.

Copyright: (C) 2022 by the authors. Licensee MDPI, Basel, Switzerland. This article is an open access article distributed under the terms and conditions of the Creative Commons Attribution (CC BY) license (https:// creativecommons.org/licenses/by/ $4.0 /)$.

\begin{abstract}
This paper discusses the influence of vehicle parameters on the identification accuracy of bridge fundamental frequency from a passing vehicle and validates such influence through laboratory tests. In the process of theoretical derivation, the interaction between a vehicle and bridge is no longer ignored to uncouple the equation group and the stepwise integration method is used to solve the equation group. Key parameters such as the vehicle speed $\alpha_{v}$, vehicle-to-bridge mass ratio $\alpha_{m v}$, and frequency ratio $\mu_{1}$, are dimensionless. Based on the simplified vehicle-to-bridge system model, the numerical analysis method is used to analyze their influence on the identified bridge frequency. Through parameter analysis, it is found that the vehicle-to-bridge mass ratio and vehicle speed have a great impact on the patterns of identified bridge frequency; and when the vehicle frequency is greater than or less than the bridge frequency, the identified bridge frequency exhibits different patterns. The bridge frequency deviation rate can be greater than $20 \%$ when the vehicle speed and vehicleto-bridge mass ratio increases. In order to ensure the accuracy of bridge frequency identification, it is recommended that the vehicle-bridge mass ratio should not exceed $10 \%$. Besides, appropriately increasing the vehicle-to-bridge mass ratio will increase the probability of accurately identifying the bridge frequency under certain conditions. In addition, a novel frequency extraction method was proposed to more accurately filter out the frequency from the vehicle acceleration spectrum.
\end{abstract}

Keywords: bridge; fundamental frequency; vehicle mass; frequency deviation; vehicle-bridge interaction; laboratory test

\section{Introduction}

Structural performance degradation is an extensively concerned issue for in-service bridges, and the inspection and evaluation is an essential and necessary work to keep bridges in good operating condition. However, a traditional bridge inspection method usually requires considerable preparatory work, such as field installation of sensors and undesirably traffic closure, which require massive time and economic costs. The disadvantages of a traditional bridge inspection method limit its wide application to highways and urban bridges. To meet the increasing maintenance requirements of these bridges, rapid detection methods and techniques are urgently needed and have received more attention in recent years [1-4].

The indirect bridge detection method was first proposed by Yang [5] as early as 2004 and has gained great popularity because of its low cost and low maintenance effort. Unlike the traditional bridge inspection method [6-8], this approach employed the dynamic response of a passing vehicle instead of a bridge itself. A vehicle acts as an exciter and a signal collection device simultaneously in this method. In the initial stage, researchers use 
the acceleration signal of a passing vehicle to extract the bridge natural frequency. The accuracy of the result was verified through a theoretical derivation $[9,10]$, laboratory investigation [11-13], and field tests [14-16]. With further development of this method, more bridge modal parameters were identified from vehicles' acceleration signal, such as modal shape and damping parameter [17-19]. In order to reduce vehicle response interference in the signal, the response of the contact point of the vehicle with the bridge, rather than the vehicle itself, is proposed [20]. On this basis, the effect of identifying bridge parameters using the acceleration signals of passing vehicles is further improved, especially the extraction of bridge frequency [21-24]. The vehicle model is also developed from a single-degree-offreedom (DOF) model to a single-axle vehicle model. The single-axle vehicle is modeled as a two DOF system, including both the rocking and vertical motions [25]. In bridge modal analysis, Malekjafarian and Obrien present a novel algorithm for the estimation of bridge mode shapes using the response measured on a passing vehicle [17]. A rescaling process is proposed to construct the bridge mode shapes using the amplitude of the responses measured on two following axles. And the concept of subtraction is employed to remove the effect of the road profile in the measured responses. They also present a novel algorithm for bridge damage detection based on the mode shapes estimated from a passing vehicle [26]. Different from previous studies, a measurement system based on laser vibrometers and accelerometers is employed to measure the bridge response at moving coordinates on a passing vehicle. The bridge mode shape is estimated with a high resolution by using an improved short time frequency domain decomposition method. Moreover, a number of signal processing methods were introduced to achieve better parameter identification by reducing false-positive feedbacks extracted from the roughness of road surfaces [27-29]. A novel indirect method of extracting bridge fundamental frequency was proposed by Wang [30], which aimed to eliminate the roughness influence in vehicle signals. A particle filter in combination with a random walk model is used to estimate the displacement inputs at the front and rear tires. Then the bridge's fundamental frequency can be clearly shown through the subtraction between the displacement inputs at the front and rear tires. Bridge damage identification methods can be divided into frequency-based and mode shape-based methods, depending on whether or not they rely on modal parameters [31]. They are both capable of damage detection $[14,32,33]$, while only the mode shape-based methods are able to realize damage localization $[19,34,35]$. In addition, signal processing and machine learning techniques, which do not rely on modal parameters, perform well in identifying structural damage location and degree [36-38].

A bridge dynamic response can be amplified by regulating the vehicle frequency, increasing vehicle weight or speed. Increasing vehicle weight becomes a feasible solution, when considering the negative effects of increasing vehicle speed and the inconvenience of altering vehicle stiffness. When analyzing the vehicle-bridge interaction (VBI) system, we usually assume that the effect of vehicle vibration on the bridge can be ignored because the vehicle weight is so small compared to that of the bridge. In Shi's paper [39], a new assumption is made to uncouple the equation group, which is that the vehicle acceleration magnitude is much lower than the gravitational acceleration constant. Although the vehicle mass is as high as $100 \%$ of the total bridge mass in Shi's research, the vehicle was maintained at a low speed in order to meet Shi's assumption. These two assumptions both ignore the interaction between the vehicle and bridge. However, this assumption tends to be invalid when the vehicle becomes heavier and the bridge becomes lighter or the vehicle speed becomes faster. In such cases, the frequency variation of the bridge coupled with the moving vehicle should be taken into account to avoid the identification deviations.

Natural frequencies are mainly sensitive to global stiffness modifications and not so much to local ones [2]. However, the global stiffness is often influenced by variations in environmental factors or heavy vehicles crossing the bridge. That influence can be high enough to completely mask the presence of even severe damage [40]. In Dimitrios's paper [41], a steel single-span tied-arch bridge was monitored for a year. An increase of $10{ }^{\circ} \mathrm{C}$ in temperature resulted in a relative decrease of about $0.2 \sim 0.25 \%$ for the natural 
frequencies of the arch modes. In the field experiment in Chang's paper [42], a decrease of $2.6 \%$ in bridge frequency was observed when a truck with mass ratio of 0.082 to the experiment bridge was parked at the midspan of the bridge. In the laboratory experiments, a decrease of $10.4 \%$ was observed when a model vehicle with mass ratio of 0.154 to the scaled bridge was parked at the midspan of the bridge. The bridge frequency also changes under daily traffic conditions. From the $24-\mathrm{h}$ ambient vibration monitoring on a cablestayed bridge, it was found that the natural frequencies of the global modes can exhibit as much as $1 \%$ variation [43]. Piotr et al. [44] showed that the natural frequencies of the bridge modes can exhibit variation between $1.7 \%$ and $5.5 \%$. When heavy vehicles pass, the frequency of simply supported Nongro bridge was approximately $5.7 \%$ larger than that when light vehicles pass [45]. Kim et al. [46] found that frequency shifts up to $5.4 \%$ for a short span and relatively high (vehicle to bridge) mass ratio. In addition, previous studies mainly discussed the variation rule of bridge frequency with different vehicle stiffness through theoretical analysis and laboratory test $[47,48]$. The analytical solution of a bridge and vehicle frequency when the vehicle is in a different position of the bridge was given, however fail to account for the applicability of bridge frequency extraction in indirect bridge detection methods.

In this paper, the equation group for the general VBI system cannot be uncoupled under the strong vehicle-bridge interaction. A strong vehicle-bridge interaction is achieved through adjusting vehicle parameters, such as a large vehicle-to-bridge mass ratio and high vehicle speed. The influence of vehicle parameters on frequency extraction from a passing vehicle is discussed in detail and the accuracy of frequency identification is demonstrated. The main parameters are made dimensionless, including vehicle speed, frequency ratio, and vehicle-to-bridge mass ratio. Euler-Bernoulli beams and a spring-mass system are used to simulate bridge and vehicle, respectively. It must be mentioned that the road surface roughness and damping coefficient are not considered in this study. In addition, a frequency extraction method was proposed to more accurately filter out the frequency from the vehicle acceleration spectrum. The proposed method is verified and discussed by the experimental tests.

\section{Vehicle-Bridge Interaction System}

The mathematical model for the VBI system is shown in Figure 1. The vehicle is modeled as a one degree-of-freedom oscillator moving over a simply-supported beam. The beam is assumed as a Euler-Bernoulli beam with constant cross-sections. Damping of the entire VBI system, and road surface roughness are also neglected. In the mathematical model, the vehicle moves along the $x$-axis of the beam to point $\mathrm{x}$ at time $t$. In Figure $1, v, k_{v}$, and $m_{v}$ is the speed, stiffness, and mass of the vehicle, respectively; $m_{b}, E I$, and $L$ is the unit mass, section stiffness, and span of the bridge, respectively; $y_{v}, y_{b}$, and $y_{w}$ is the vertical displacement of vehicle, bridge, and wheel, respectively; and $g$ is the for acceleration due to gravity. The equations of motion at time $t$ for the vehicle and bridge can be written each as follows,

$$
\begin{gathered}
m_{v} \ddot{y}_{v}+k_{v}\left(y_{v}-y_{b, x=v t}\right)=0 \\
m_{b} \frac{\partial^{2} y_{b}(x, t)}{\partial t^{2}}+E I \frac{\partial^{4} y_{b}(x, t)}{\partial x^{4}}=f_{N} \delta(x-v t)
\end{gathered}
$$

where $f_{N}=m_{v}\left(g-\ddot{y}_{v}\right)+m_{w}\left(g-\ddot{y}_{w}\right)$. 


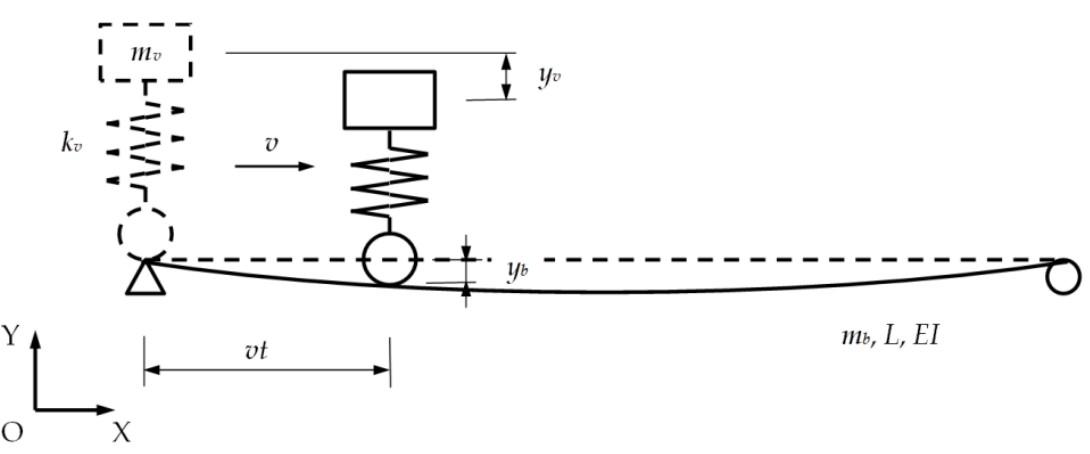

Figure 1. The mathematical model for the vehicle-bridge interaction (VBI) system.

The assumptions made in this study are: 1. The bridge follows Hooke's law, Navier's hypothesis, Saint-Venant's principle, and small-deflection theory; 2 . the wheels of the vehicle stay on the deck constantly while it passes through the bridge; 3 . the bridge is initially at rest before the vehicle starts; and 4 . the vehicle speed and its path are constant numbers and the suspension spring is considered to be linearly elastic.

By making the above equation dimensionless, the dimensionless frequencies of vehicles and bridges can be expressed as:

$$
\begin{gathered}
\Omega_{v}=\sqrt{\frac{m_{b}}{E I}} L^{2} \omega_{v} \\
\Omega_{b n}=\sqrt{\frac{m_{b}}{E I}} L^{2} \omega_{b n}=\sqrt{\frac{m_{b}}{E I}} L^{2}\left(\frac{n \pi}{L}\right)^{2} \sqrt{\frac{E I}{m_{b}}}=(n \pi)^{2} .
\end{gathered}
$$

Using modal superposition, $\alpha_{y b}(s, \tau)=\sum_{n=1}^{\infty} H_{n}(\tau) \sin (n \pi s)$, where $\sin (n \pi s)$ and $H_{n}(\tau)$ is the $n$-th mode shape and mode displacement, respectively. Substituting the aforementioned expressions into Equations (1) and (2), multiplying both sides of Equation (2) by $\sin (n \pi s)$ and integrating $s$ from 0 to 1 , we obtain:

$$
\begin{gathered}
\ddot{H}_{m}(t)+2 \alpha_{m w} \sum_{n=1}^{\infty} \ddot{H}_{n}(\tau) \sin (n \pi s) \sin (m \pi s)+4 \alpha_{m w} \alpha_{v} \sum_{n=1}^{\infty}(n \pi) \dot{H}_{n}(\tau) \cos (n \pi s) \sin (m \pi s) \\
+\Omega_{b m}^{2} H_{m}(\tau)-2 \alpha_{m w} \alpha_{v}^{2} \sum_{n=1}^{\infty}(n \pi)^{2} H_{n}(\tau) \sin (n \pi s) \sin (m \pi s) \\
+2 \alpha_{m v} \Omega_{v}^{2} \sum_{n=1}^{\infty} H_{n}(\tau) \sin (n \pi s) \sin (m \pi s)-2 \alpha_{m v} \Omega_{v}^{2} \alpha_{y v} \sin (m \pi s)=2\left(\alpha_{m v}+\alpha_{m w}\right) g^{*} \sin (m \pi s) \\
\frac{\partial^{2} \alpha_{y v}}{\partial \tau^{2}}+\Omega_{v}^{2} \alpha_{y v}-\Omega_{v}^{2} \sum_{n=1}^{\infty} H_{n}(\tau) \sin (n \pi s)=0
\end{gathered}
$$

where, $\alpha_{y b}, \alpha_{y v}, \alpha_{m v}, \alpha_{m w}, \alpha_{v}, s, \tau$, and $g^{*}$ are the dimensionless parameters of $y_{b}, y_{v}, m_{v}$, $m_{w}, v, x, t$, and $g$, respectively. Their expressions are detailed in Appendix A. The above equations can be solved by using the stepwise integration method.

It is generally believed that the mass of the vehicle is far less than the mass of the bridge, which means the impact of the vehicle vibration on bridge vibration is ignored. Thus, we have $m_{v} \ll m_{b} L, m_{w} \ll m_{b} L$, namely $\alpha_{m v} \rightarrow 0, \alpha_{m w} \rightarrow 0$. Equation (5) can be simplified as:

$$
\ddot{H}_{m}(\tau)+\Omega_{b m}^{2} H_{m}(\tau)=2\left(\alpha_{m v}+\alpha_{m w}\right) g^{*} \sin (m \pi s) .
$$

Under zero initial conditions, we obtain:

$$
H_{m}(\tau)=\frac{2\left(\alpha_{m v}+\alpha_{m w}\right) g^{*}}{\Omega_{b m}^{2}\left(1-S_{m}^{2}\right)}\left[\sin (m \pi s)-S_{m} \sin \left(\Omega_{b m} \tau\right)\right]
$$


where $S_{m}=\frac{n \pi \alpha_{v}}{\Omega_{b m}}$.

Substituting Equation (8) into Equation (6), vehicle response $\alpha_{y v}$ can be solved from Equation (6) by Duhamel's integral. Then, $\alpha_{y v}$ can be differentiated twice to yield the acceleration of the vehicle as:

$$
\ddot{\alpha}_{y v}=A_{1} \cos \Omega_{v} \tau+A_{2} \cos 2 n \pi s+A_{3} \cos \left(\Omega_{b n}-n \pi \alpha_{v}\right) \tau+A_{4} \cos \left(\Omega_{b n}+n \pi \alpha_{v}\right) \tau
$$

where, $\mu_{n}=\Omega_{v} / \Omega_{b n}$ is the ratio of vehicle frequency to bridge frequency, in which $n$ represents the $n$-th frequency of the bridge. The expressions of $A_{1}, A_{2}, A_{3}$, and $A_{4}$ are detailed in Appendix B.

It can be seen from Equation (9), the acceleration of the vehicle $\ddot{\alpha}_{y v}$ is mainly composed of four parts, including vehicle frequency term $\Omega_{v}$, bridge frequency term $\Omega_{b n} \pm \pi \alpha_{v}$, and speed term $2 n \pi \alpha_{v}$. Apparently, the bridge frequency derived from Equation (9) is $\Omega_{b n} \pm \pi \alpha_{v}$, which is only related to the speed term. The frequency deviation rate can be defined as:

$$
\Delta_{b}=\frac{\Omega_{b e x}-\Omega_{b n}}{\Omega_{b n}}
$$

where $\Omega_{b e x}$ represents the bridge frequency identified through vehicle acceleration. When the identified bridge frequency is less than the corresponding bridge frequency, it is called the left-biased frequency, corresponding to $\Delta_{b}<0$. On the contrary, it is called the rightbiased frequency, corresponding to $\Delta_{b}>0$. Therefore, disregarding vehicle mass, the bridge frequency deviation rate is:

$$
\left|\Delta_{b}\right|=\left|\frac{\Omega_{b e x} \pm \Omega_{b 1}}{\Omega_{b 1}}\right|=\frac{\pi \alpha_{v}}{\Omega_{b 1}} .
$$

As mentioned in previous research results [5], the value of $\Delta_{b}$ is only related to vehicle speed. In summary, the vehicle speed is positively related to the frequency deviation rate $\Delta_{b}$.

\section{Peak Frequency Identification Method}

Judgment principles need to be defined primarily on how to identify the fundamental frequency of the bridge from the acceleration spectrum of the vehicle. The central idea is to select the peaks worth paying attention to according to specific indicators from the many peaks in the spectrogram within a specific frequency range. Then, known peaks are removed from the selected peaks and result in the isolation of the target peak. Combined with the issue at hand, the fundamental problem is to select the vehicle frequency item, bridge frequency item, and speed item from the spectrogram. When the vehicle frequency item and speed item are eliminated, the bridge frequency can be obtained.

Firstly, the following parameters are introduced: Peak amplitude $F_{p v}$, peak aspect ratio $F_{h w}$, peak area $F_{a e}$, and peak significance $F_{s r}$. Their expressions are explained in the following section and in Figure 2.

$$
\begin{gathered}
F_{p v}(i)=Y_{i}^{\max } \\
F_{a e}(i)=\frac{1}{2}\left[Y_{i-1}^{\min }+Y_{i+1}^{\min }\right] Y_{i}^{\max } \\
F_{h w}(i)=\frac{Y_{i}^{\max }-\frac{Y_{i-1}^{\min }+Y_{i+1}^{\min }}{2}}{X_{i+1}^{\min }-X_{i-1}^{\min }} \\
F_{s r}(i)=\min \left(\frac{Y_{i}^{\max }}{Y_{i-1}^{\min }}, \frac{Y_{i}^{\max }}{Y_{i+1}^{\min }}\right)
\end{gathered}
$$

where, $X_{i}^{\max }$ or $X_{i}^{\min }$ represents the frequency value corresponding to the $i$-th extreme point and $Y_{i}^{\max }$ or $Y_{i}^{\min }$ represents the amplitude of the $i$-th extreme point. The superscript max 
and min indicate the maximum value and minimum value, respectively. The physical meaning of $F_{h w}$ is a quantity describing the peak shape. The larger the value of $F_{h w}$, the more concentrated the frequency corresponding to the peak. $F_{a e}$ and $F_{s r}$ describe the ease in which the peak can be identified. The larger their value, the easier the peak is to be observed. $F_{p v}$ indicates the degree of concern for the peak. Generally, it can be considered that the corresponding frequency with a large value of $F_{p v}$ is the main component of the signal.

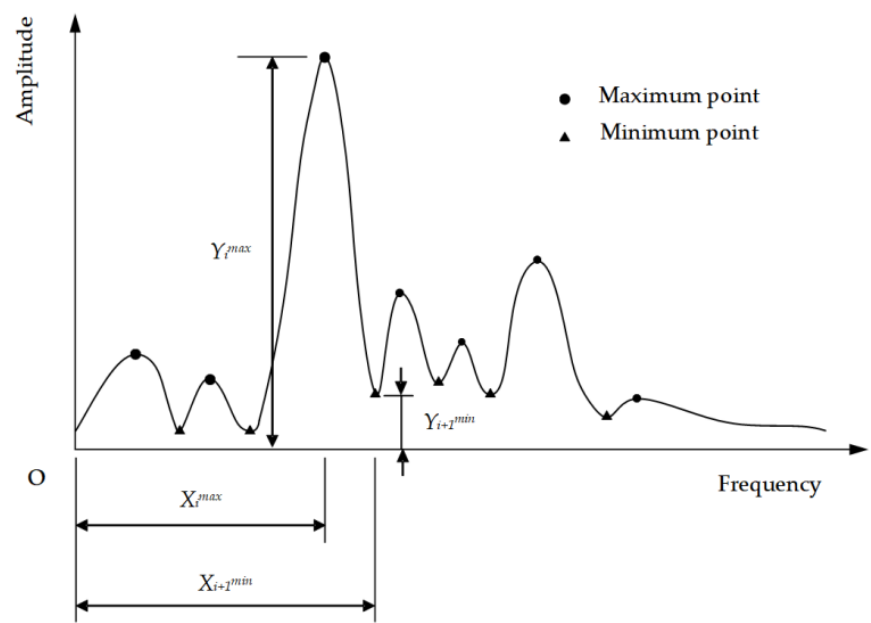

Figure 2. Schematic diagram of peak parameters.

Suppose there are $n$ maximum points in the frequency range of interest, so the basic score of each maximum point can be defined as:

$$
V_{F B}(i)=F_{h w}(i)+F_{s t}(i)+F_{a e}(i) / \max \left(F_{a e}(k)\right)
$$

where $k=1,2, \cdots \cdots, n$.

In order to improve identification accuracy of the peaks, it is necessary to make the following supplements:

1. To ensure the significance of the maximum point between two adjacent minimum points, it is recommended that the amplitude of the maximum point should be at least $10 \%$ greater than that of the adjacent minimum point, which means $F_{s r}(i)>1.1$.

2. In order to ensure the visibility of peaks, the area corresponding to each peak should not be too low. The ratio of the area corresponding to the peak in the same spectrogram to the maximum area should not be too low. To be conservative, take $F_{a e}(i) / \max \left(F_{a e}(k)\right)>0.01$ in this paper. Thus, the peak discrimination function is defined as:

$$
W_{p i}(i)=\left\{\begin{array}{ll}
1 & F_{s r}(i)>1.1 \quad \text { or } \quad F_{a e}(i) / \max \left(F_{a e}(k)\right)>0.01 \\
0 & \text { else }
\end{array} .\right.
$$

A special case, as shown in Figure 3, has multiple successively decreasing small peaks (boxed in red) on the side of a major peak. This situation becomes more prominent as the vehicle crossing the bridge slows down, the number of sampling points increases, and the vehicle-to-bridge mass ratio increases. Preliminary analysis indicates that this phenomenon is caused by real-time changes in the frequency of vehicles and bridges in the VBI system as vehicles pass through the bridge. Applying Fast Fourier transformation alone to the signal cannot satisfactorily reflect the real-time frequency change in the spectrogram. 


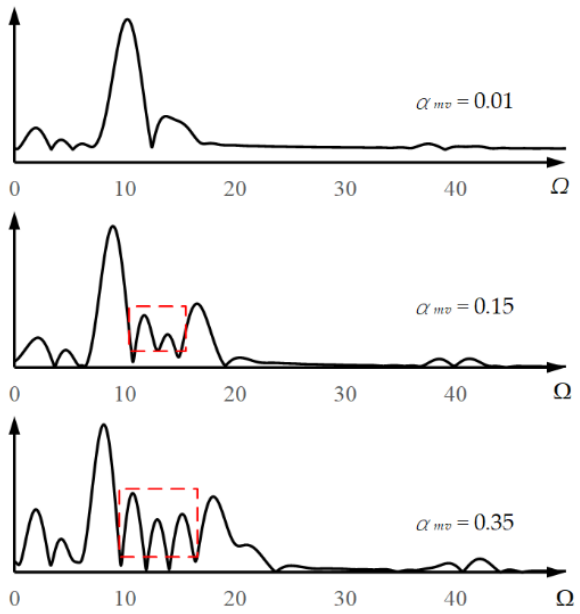

(a)

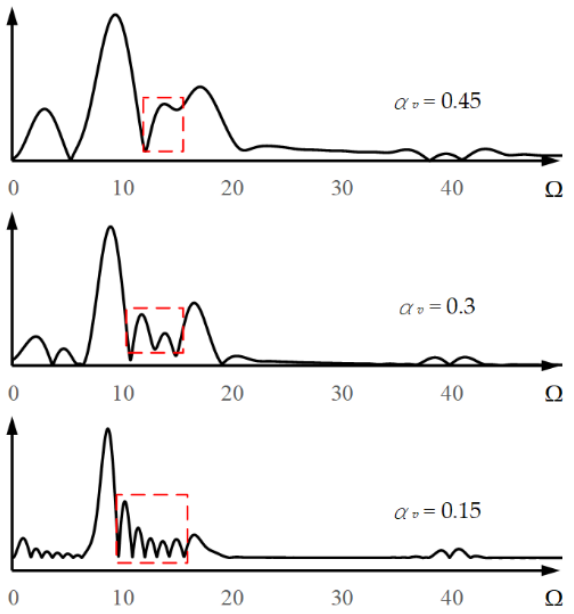

(b)

Figure 3. Vertical acceleration spectrum of vehicle with different (a) $\alpha_{m v}$ and (b) $\alpha_{v}$.

For illustrative purposes, two cases are demonstrated briefly. The value of each parameter is shown in Table 1. The Hilbert transform was used to analyze the vehicle and bridge terms in the vehicle acceleration signal, as well as to obtain their time-frequency curve during the passage of the vehicle on the bridge, shown in Figure 4.

Table 1. Value of parameters.

\begin{tabular}{cccccc}
\hline & Case 1 & & Case 2 \\
\hline$\mu_{1}$ & & 1.5 & & 1.5 & \\
$\alpha_{m v}$ & 0.01 & 0.15 & 0.35 & 0.15 & 0.45 \\
$\alpha_{v}$ & & 0.3 & & 0.15 & 0.3 \\
\hline
\end{tabular}

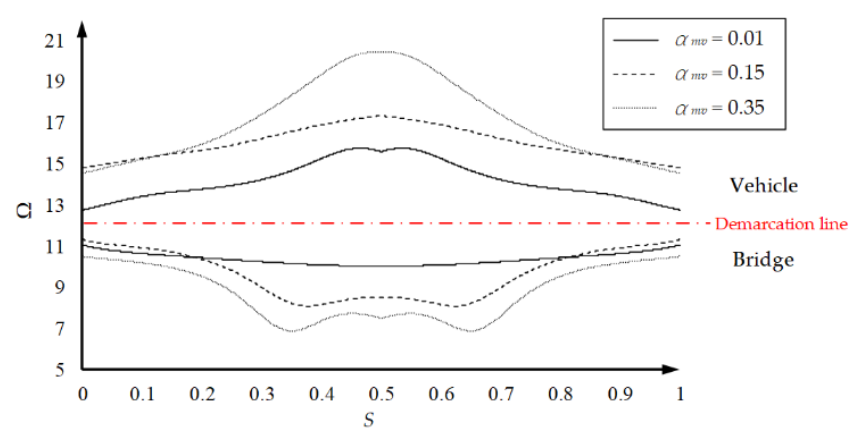

(a)

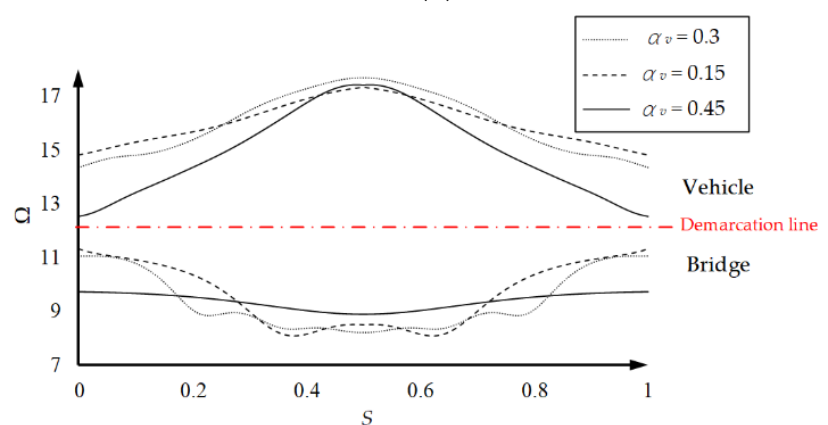

(b)

Figure 4. Frequency variation of vehicle and bridge when vehicle passing the bridge. (a) With different vehicle mass; (b) with different speed. 
In order to minimize the interference of these small peaks and highlight the frequency of the vehicle and bridge, gain factors are defined for each major peak. The approach of assigning gain coefficient is as follows: (1) The top three with the largest value of $F p v$ are given 5,2 , and 1 in turn:

$$
W_{G 1}(i)=\left\{\begin{array}{ll}
5 & \operatorname{Rank}\left(F_{p v}(i)\right)=1 \\
2 & \operatorname{Rank}\left(F_{p v}(i)\right)=2 \\
1 & \text { else }
\end{array} .\right.
$$

For the maximum point in the maximum point, assign 5 :

$W_{G 2}(i)=\left\{\begin{array}{ll}5 & F_{p v}(i) \text { is the maximum point among the maximum points } \\ 1 & \text { else }\end{array}\right.$.

In summary, the score of the maximum point is:

$$
V_{F}(i)=V_{F B}(i) \times W_{p i}(i) \times \max \left(W_{G 1}(i), W_{G 2}(i)\right) .
$$

Peaks in the spectrogram were sorted according to the above scoring principle. The acceleration of the vehicle contains the vehicle frequency term, bridge frequency term, and speed term, which correspond to the vehicle frequency, the bridge frequency, and the speed frequency in the vehicle vertical acceleration spectrum, respectively. In this paper, most of the top three scoring peaks correspond to the bridge frequency, vehicle frequency, and speed frequency. The intuitive performance on the spectrogram is three significant peaks. After excluding the vehicle frequency $\Omega_{v e x}$ and speed frequency $\Omega_{s e x}$ from these three frequencies, the resulting bridge frequency $\Omega_{b e x}$ can be obtained successfully. However, changes in the vehicle-to-bridge mass ratio $\alpha_{m v}$, vehicle-to-bridge frequency ratio $\mu_{1}$, and vehicle speed $\alpha_{v}$, pose difficulties in bridge frequency recognition.

1. When $\alpha_{m v}$ is small and $\alpha_{v}$ is large, the vehicle-bridge interaction is weak. At the same time, the number of collection points is small. Although $\Omega_{\text {vex }}$ can be identified, the bridge frequency term cannot be fully reflected in the vehicle acceleration signal, which leads to the failure of extracting $\Omega_{b e x}$.

2. When the vehicle speed gradually increases to meet $\alpha_{v}>2 \pi \Omega_{v}$, which is $L / v<$ $1 / 2 \pi \omega_{v}$, the time for the vehicle to cross the bridge is less than its vibration period. The vehicle has left the bridge before finishing a complete vibration cycle. This will result in that the vehicle acceleration signal does not include the vehicle frequency term, and $\Omega_{\text {vex }}$ cannot be obtained.

3. When $\mu_{1}$ is small, the vehicle frequency is too low to distinguish between vehicle frequency and speed terms in the spectrogram.

4. The vehicle frequency coincides with the high-order frequency of the bridge. While the overlap between the vehicle frequency and bridge fundamental frequency is avoided in this paper, the vehicle frequency term coincides with the $n$-th order bridge frequency term in the spectrogram when $\mu_{1}$ is close to $n^{2}(n>2)$. As a result, it is impossible to solve for $\Omega_{v e x}$, however it generally does not affect the identification of $\Omega_{b e x}$.

In summary, $\Omega_{b e x}$ is calculated corresponding to the highest scoring peak excluding $\Omega_{v e x}$ and $\Omega_{\text {sex }}$. Alternatively, the frequency corresponding to the highest peak can be temporarily interpreted as the bridge frequency $\Omega_{b e x}$ until further verification is conducted provided with the values of each parameter.

\section{The Vehicle-to-Bridge Mass Ratio on Frequency Deviation Rate \\ 4.1. Numerical Examples}

The frequency of the vehicle is generally between $0 \sim 15 \mathrm{~Hz}$ in reality [2,49]. Based on the numerical examples in Yang's study [5], the range of each dimensionless parameter is as follows: $\mu_{1}=0.2 \sim 0.9,1.2 \sim 15, \alpha_{m v}=0.01 \sim 0.5, \alpha_{v}=0.03 \sim 0.75$, and $\alpha_{w}=0.001$. 
Substituting the above parameters into Equations (5) and (6), the acceleration time history curve of vehicles passing the bridge can be obtained. Then Fast Fourier transform can be used to obtain the vehicle acceleration spectrum. Using the method described Section 3, the dimensionless bridge fundamental frequency can be identified, and then the corresponding frequency deviation rate can be obtained using Equation (10). The main results of the numerical calculation are shown in Figures 5 and 6, and the shaded part in the figure indicates that the recognition failed. The colors in Figures 5 and 6 gradually change from blue to red, representing the gradual change of the bridge fundamental frequency deviation rate from the minimum value to the maximum value. Blue and red represent the minimum and maximum values in their respective graphs. The different colors indicate the relative magnitude of the bridge fundamental frequency deviation rate in each graph. Therefore, the absolute values of the bridge fundamental frequency deviation rate represented by the colors in different pictures are not the same.

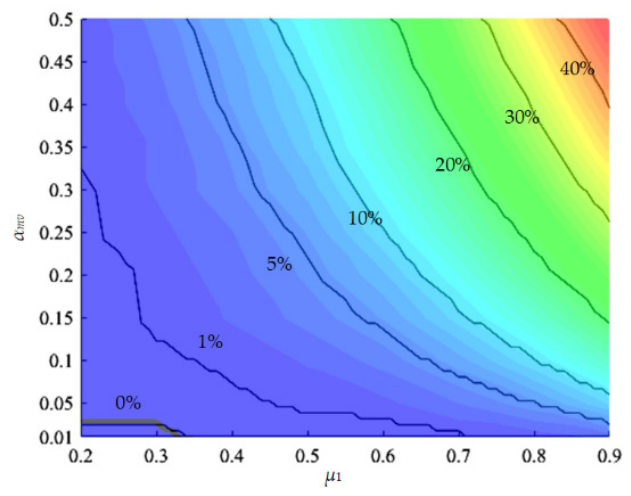

(a)

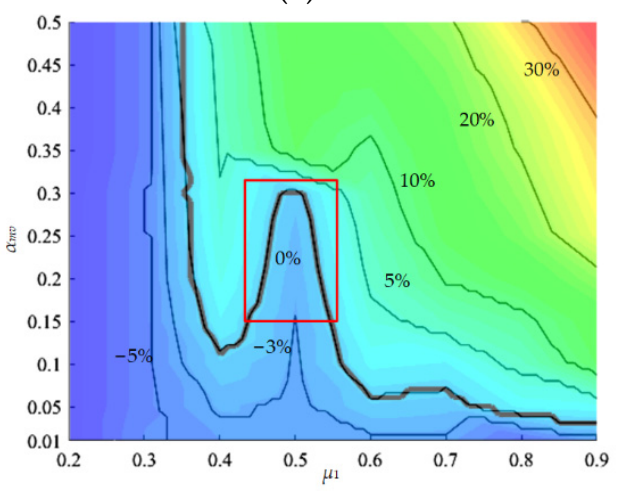

(c)

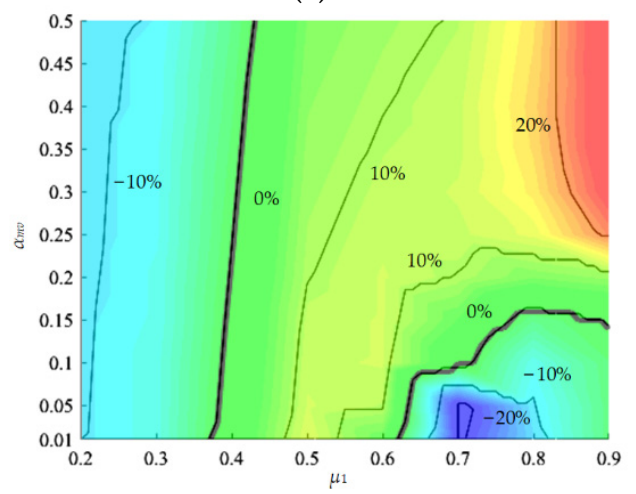

(e)

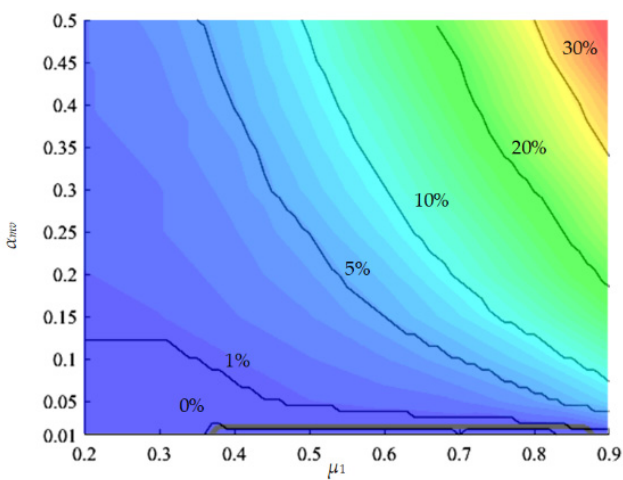

(b)

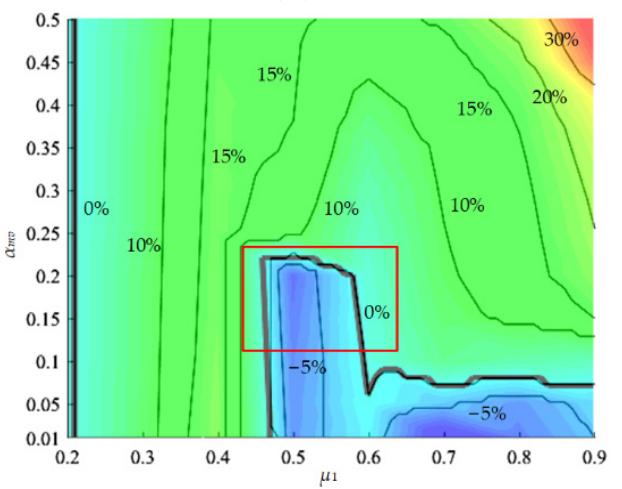

(d)

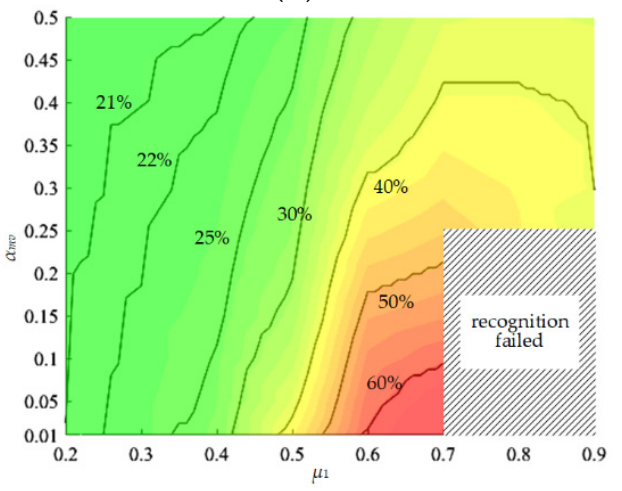

(f)

Figure 5. Bridge fundamental frequency deviation rate $\left(\mu_{1}<1\right)$. (a) $\alpha_{v}=0.03$, (b) $\alpha_{v}=0.15$, (c) $\alpha_{v}=0.30$, (d) $\alpha_{v}=0.45,(\mathbf{e}) \alpha_{v}=0.60,(\mathbf{f}) \alpha_{v}=0.75$. 


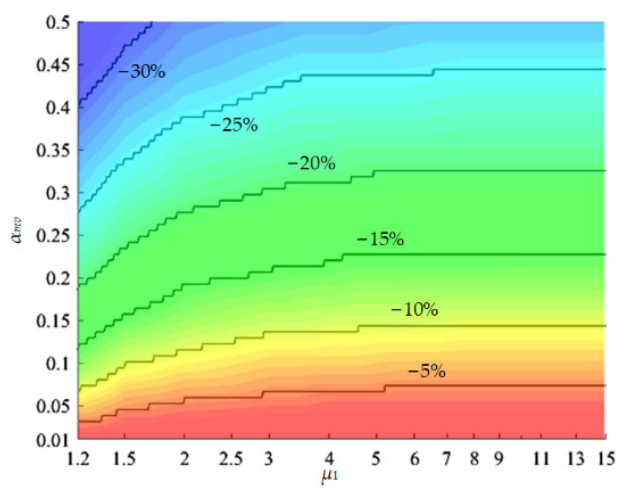

(a)

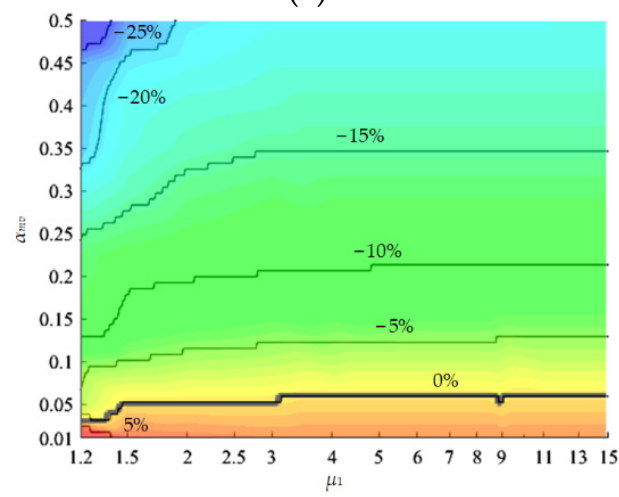

(c)

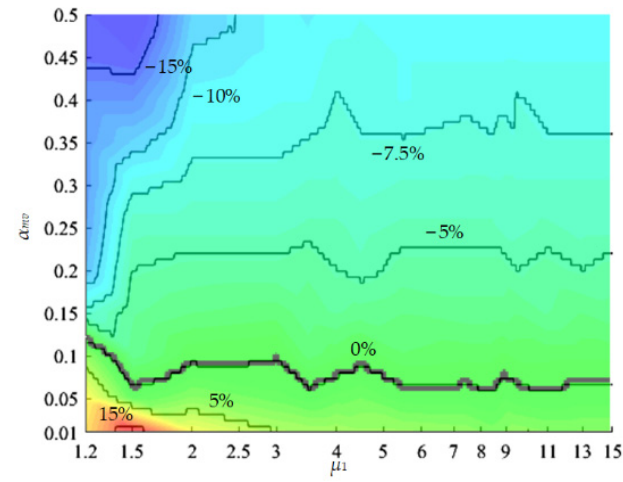

(e)

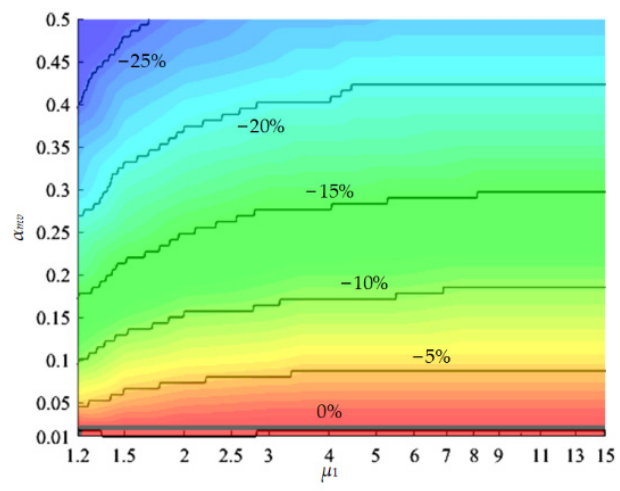

(b)

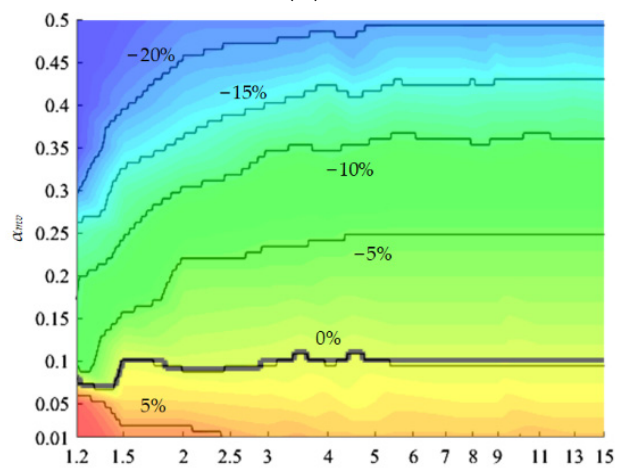

(d)

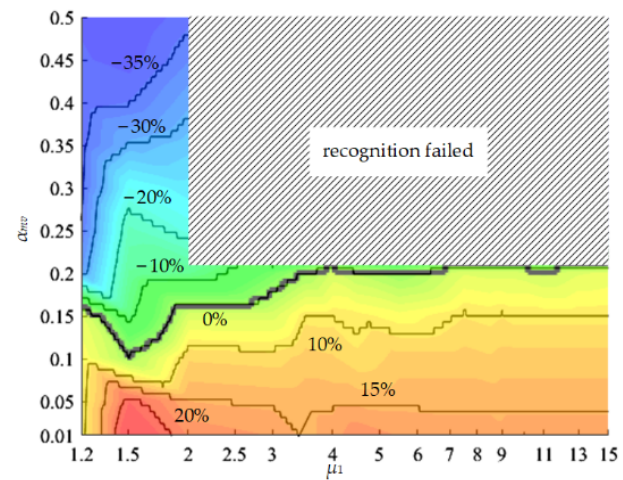

(f)

Figure 6. Bridge fundamental frequency deviation rate $\left(\mu_{1}>1\right)$. (a) $\alpha_{v}=0.03$, (b) $\alpha_{v}=0.15$, (c) $\alpha_{v}=0.30$, (d) $\alpha_{v}=0.45,(\mathbf{e}) \alpha_{v}=0.60$, (f) $\alpha_{v}=0.75$.

\subsection{Results Description}

Without considering the mass of the vehicle, the bridge fundamental frequency deviation rate $\left|\Delta_{b}\right|$ is $0.72 \%, 3.6 \%, 7.2 \%, 10.8 \%, 14.4 \%$, and $18.0 \%$, their corresponding value of $\alpha_{v}$ is $0.03,0.15,0.30,0.45,0.60$, and 0.75 , respectively. However, as shown in Figures 5 and 6 even when $\alpha_{m v}$ is 0.01 , the $\Omega_{b e x}$ will change with the change of $\mu_{1}$, and both the left-biased and right-biased frequencies will appear. Therefore, it should be noted that introducing vehicle mass also brings the influence of the vehicle-to-bridge frequency ratio.

As shown in Figures 5 and 6, at different speeds (take $\alpha_{v}$ as 0.03, 0.15, 0.30, 0.45, 0.60, and 0.75 , respectively) $\Delta_{b}$ varies with $\mu_{1}$ and $\alpha_{m v}$ in inconsistent patterns.

In the case of $\mu_{1}>1$, the recognition accuracy becomes increasingly worse with the increase of $\alpha_{v}$. When $\alpha_{v}$ is small $\Delta_{b}<0$, and $\Delta_{b}$ increases with the increase of $\mu_{1}$ and gradually stabilizes. As $\alpha_{v}$ increases, $\Delta_{b}$ will become greater than zero when $\alpha_{m v}$ and $\mu_{1}$ are small, and the range of this situation becomes larger. $\Delta_{b}$ gradually decreases as $\mu_{1}$ increases 
under the condition of $\Delta_{b}<0$. Generally speaking, when $\mu_{1}>1, \Omega_{b e x}$ is mainly left-biased frequency and $\left|\Delta_{b}\right|$ increases as $\alpha_{v}$ and $\mu_{1}$ decrease. Specifically, $\Delta_{b}$ decreases as $\alpha_{v}$ and $\alpha_{m v}$ increase when $\mu_{1}$ is constant, and $\Delta_{b}$ decreases as $\alpha_{m v}$ increase when $\alpha_{v}$ is constant.

Compared to the case where $\mu_{1}$ is greater than 1 , the changing pattern of $\Delta_{b}$ is relatively complicated when $\mu_{1}$ is less than 1 . Similar to the previous case, as $\alpha_{v}$ increases, the recognition accuracy becomes increasingly worse. As shown in Figure 4, the shaded part indicates that the fundamental frequency of the bridge cannot be identified. When $\alpha_{v}$ is small, $\Delta_{b}>0$. There will be a situation where $\Delta_{b}$ is less than zero when $\alpha_{m v}$ or $\mu_{1}$ is small as $\alpha_{v}$ increases. Especially, within specific range of $\mu_{1}$, the area where $\Delta_{b}<0$ will extend to where the value of $\alpha_{m v}$ is comparatively large (as shown in the red frame of Figure 5c,d). Generally speaking, $\Omega_{b e x}$ is mainly a right-biased frequency when $\mu_{1}<1$. Specifically, as $\alpha_{m v}$ and $\alpha_{v}$ increases, $\Delta_{b}$ increases and decreases, respectively when $\mu_{1}$ is constant, however when $\alpha_{v}$ continues to increase, the above pattern shows the opposite trend. In most cases, $\Delta_{b}$ increases as $\mu_{1}$ increases while $\alpha_{m v}$ remains constant, however the pattern is different in the area where $\Delta_{b}<0 ; \Delta_{b}$ increases as $\mu_{1}$ and $\alpha_{m v}$ increases while $\alpha_{v}$ remains constant, however decreases when $\alpha_{v}$ becomes larger.

\subsection{Probability of Accurately Identifying Bridge Frequencies}

Here, we define that when $\left|\Delta_{b}\right|<0.05$, the bridge fundamental frequency is considered to be accurately identified. Since vehicle speed and mass mentioned in the above analysis process are accurately known, they are considered as controllable parameters. Within the specified range of parameters in the previous section, $\alpha_{m v}$ and $\alpha_{v}$ are selected as variables. The probability of accurately identifying the bridge fundamental frequency was obtained, as shown in Figure 7. In Figure 7a, when $\alpha_{m v}<0.05$ and $\alpha_{v}<0.3$, the probability of accurately identifying the bridge fundamental frequency is greater than $80 \%$. However, the probability of the bridge fundamental frequency being accurately identified is less than $50 \%$ when $\alpha_{m v}>0.25$. If the standard for accurately identifying fundamental bridge frequencies is $\left|\Delta_{b}\right|<0.1$ and the results are shown in Figure $7 \mathrm{~b}$. It can be seen from Figure $7 \mathrm{~b}$ that the resulting probability has increased significantly. The probability of the bridge fundamental frequency being accurately identified is greater than $80 \%$ when $\alpha_{m v}<0.1$ and $\alpha_{v}<0.6$. From the conclusion drawn from Figure 7, when $0.3<\alpha_{v}<0.6$ and $\alpha_{m v}<0.1$, selecting the value of $\alpha_{m v}$ carefully can improve the probability of accurately identifying the bridge fundamental frequency to a certain extent. To ensure the accuracy of identifying the bridge fundamental frequency, the vehicle-to-bridge mass ratio should not be selected as too high and the vehicle speed should not be too fast. If either of them is too large, it will cause a large deviation in the identified frequency.

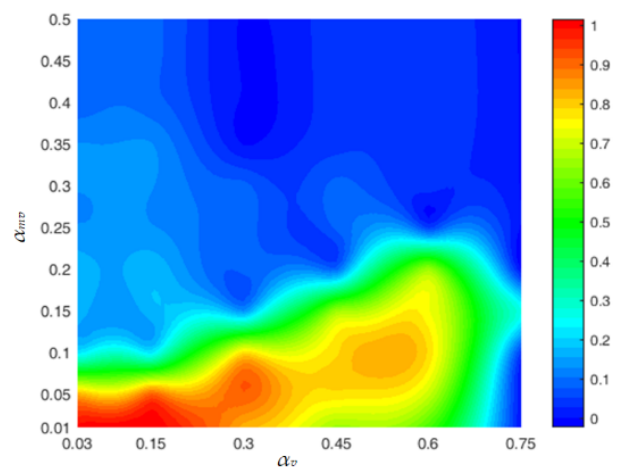

(a)

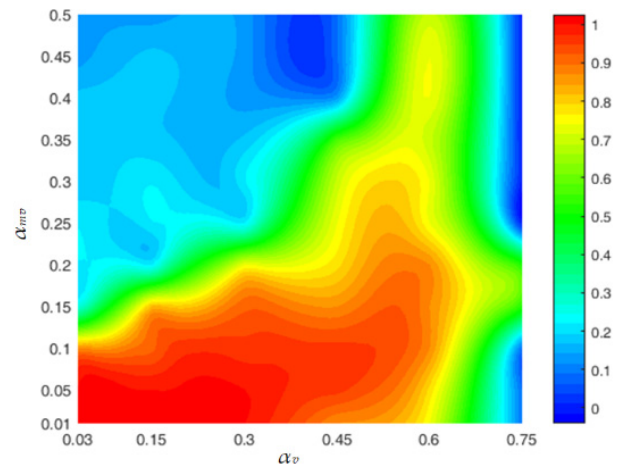

(b)

Figure 7. Probability of identifying bridge fundamental frequencies. (a) $\left|\Delta_{b}\right|<0.05$, (b) $\left|\Delta_{b}\right|<0.1$.

In the parameter analysis, in order to avoid resonance between the vehicle and bridge, $\mu_{1}$ is not selected as a value between 0.9 and 1.2. However, for short and rigid bridges, when vehicles move at a relatively low speed on the bridge, vehicle frequencies may overlap 
with the bridge frequencies. Faced with this problem, the most direct way is to change the frequency of vehicles or bridges so that the two are no longer close. Obviously, for bridges that must be used continuously, as only the frequency of the vehicles can be adjusted. Adjusting the frequency of the vehicle can be accomplished by increasing or decreasing the vehicle's mass, or by changing the vehicle's suspension stiffness. In comparison, the method of changing vehicle mass is relatively simple. In order to make the frequency change obvious, it is recommended that the adjusted vehicle mass should not be less than 2 times the original mass or more than half of the original mass. After adjusting the vehicle frequency, try to identify the bridge frequency again.

It should be noted that due to the limitation of the simplified VBI model, the results of the numerical simulation in this paper should not be directly applied to the actual bridge. In addition, for the actual bridge, the value of $\alpha_{v}$ may not reach the upper limit of the parameter analysis. Different bridges have different $\alpha_{v}$ value ranges. Therefore, for vehicle speed, we can only give advice not to be too fast. For large-span bridges, due to the large self-weight, the problem of the obvious shift in identified frequency is generally not considered. Instead, the focus should be on how to obtain a stronger bridge response. For small-span bridges or bridges with small self-weight, attention should be paid to the frequency deviation when the vehicle mass is large or there are heavy vehicles, such as buses or trucks, passing through the bridge. Therefore, it is recommended that the vehicleto-bridge mass ratio $\alpha_{m v}$ be less than 0.1 , or even 0.05 , to ensure the success of accurately identifying bridge frequencies.

\subsection{Parameters Sensitivity Analysis}

The Morris method is used to qualitatively analyze the effect of the vehicle-bridge mass ratio $\alpha_{m v}$, vehicle-bridge frequency ratio $\mu_{1}$, and vehicle speed $\alpha_{v}$ on the bridge frequency deviation rate $\Delta_{b}$. The Morris method was first proposed by Max D. Morris in 1991 [50]. It is a discrete search method based on parameter space, which studies model parameters on a global scale. Its main idea is to assume elementary effects $(E E)$ that measure the sensitivity of the parameter $x_{i}$ obeys probability distribution $f_{i}$, and the global sensitivity of the parameter $x_{i}$ can be determined by measuring the mean and standard deviation of the distribution. If the mean value of the parameter $x_{i}$ is larger, it has a greater impact on the model output. The standard deviation $\sigma$ is used to measure the interaction between parameters. The larger the value, the greater the interaction and vice versa.

For a model with $k$ parameters, assume that the range of parameters is $[0,1]$. For a given parameter vector:

$$
X=\left(x_{1}, x_{2}, \cdots, x_{k}\right) .
$$

where each $x_{i}$ may take on value $\left\{0, \frac{1}{p-1}, \frac{2}{p-1}, \cdots, 1\right\}$ and $p$ is the number of levels. The elementary effect of the $i$-th input is calculated by the formula below:

$$
E E_{i j}=\frac{Y\left(x_{1}, \cdots, x_{j}+\Delta_{j}, \cdots, x_{k}\right)-Y(X)}{\Delta_{j}}
$$

where $Y(X)$ is the model output corresponding to the initial parameter vector and $Y\left(x_{1}, \cdots, x_{j}+\Delta_{j}, \cdots, x_{k}\right)$ is the model output after the $j$-th parameter of the initial parameter vector is changed; $\Delta$ is the magnitude of the step between 0 and $1-1 /(p-1)$, which is a multiple of $1-1 /(p-1)$. To calculate $E E$ of the remaining $k-1$ parameters, the above procedure is repeated. Then, randomly generate $n$ initial vectors and repeat the above process to calculate the $n$ elementary effects of the $k$ parameters, which is $E E_{i j}(j=1,2, \cdots k \quad i=1,2, \cdots n)$. The mean and standard deviation of $E E_{i j}$ can be expressed as:

$$
\mu_{i}=\sum_{j=1}^{n} E E_{i j} / n
$$




$$
\sigma_{i}=\sqrt{\sum_{j=1}^{n}\left(E E_{i j}-\mu_{i}\right)^{2} / n} .
$$

Combined with the actual situation, the frequency of the vehicle is generally between $0 \sim 15 \mathrm{~Hz}[2,49]$. Therefore, during parameter analysis, the value range of each dimensionless parameter are $\mu_{1}=0.2 \sim 0.9,1.2 \sim 15, \alpha_{m v}=0.01 \sim 0.5$, and $\alpha_{v}=0.03 \sim 0.75$. Take the identified bridge fundamental frequency increment $\Delta_{b}$ as the model output. There are three variables, and the parameter space is divided into five equal parts. The sampling calculation results are shown in Table 2 to discuss the global sensitivity of each parameter by using the Morris method.

Table 2. Parameters' sensitivity analysis results.

\begin{tabular}{|c|c|c|c|c|c|c|}
\hline & \multicolumn{3}{|c|}{$\mu_{1}<1$} & \multicolumn{3}{|c|}{$\mu_{1}>1$} \\
\hline & $\mu_{1}$ & $\alpha_{m v}$ & $\alpha_{v}$ & $\mu_{1}$ & $\alpha_{m v}$ & $\alpha_{v}$ \\
\hline$\mu$ & $5.650 \times 10^{-1}$ & $4.258 \times 10^{-1}$ & $5.417 \times 10^{-1}$ & $5.333 \times 10^{-2}$ & $6.200 \times 10^{-1}$ & $3.625 \times 10^{-1}$ \\
\hline$\sigma$ & $3.180 \times 10-1$ & $5.106 \times 10^{-1}$ & $4.010 \times 10^{-1}$ & $3.409 \times 10^{-3}$ & $2.378 \times 10^{-1}$ & $2.251 \times 10^{-2}$ \\
\hline
\end{tabular}

It can be seen from Table 2 that when $\mu_{1}>1, \alpha_{m v}$ and $\alpha_{v}$ have great impact to $\Delta_{b}$. Moreover, the interaction between $\mu_{1}$ and the other two parameters is not obvious. When $\mu_{1}<1, \mu_{1}, \alpha_{m v}$, and $\alpha_{v}$ all have a great impact on $\Delta_{b}$. There is also a similar degree of interaction between them. The above conclusion is mutually verified with the pattern described in the previous section. For instance, when $\mu_{1}>1$, the value of $\Delta_{b}$ tends to be stable as $\mu_{1}$ increases; when $\mu_{1}<1$, the rule of change of $\Delta_{b}$ caused by changing the values of $\mu_{1}$ and $\alpha_{m v}$ will also change accordingly as $\alpha_{v}$ takes on a different value.

\section{Laboratory Test Verification}

The test uses steel beams to simulate the bridge, and the design frequency of steel beams is about $7 \mathrm{~Hz}$. The design drawing of the steel beam is shown in Figure 8. The steel beam is made of 3-mm thick steel plate, which is $3 \mathrm{~m}$ long and $30 \mathrm{~cm}$ wide. The steel plate is equipped with 2 longitudinal ribs and 5 transverse ribs The ribs are made of iron slats with a width of $3 \mathrm{~cm}$ and a thickness of $3 \mathrm{~mm}$. Two longitudinal ribs are arranged symmetrically with an interval of $15 \mathrm{~cm}$, and 5 transverse ribs are respectively arranged at the end, 1/4 span, and mid-span of the beam. Dimensions of the support is shown in Figure 8 The finite element model of the steel beam is established by using shell elements. The first three vertical frequencies of the steel beam are $7.00 \mathrm{~Hz}, 25.24 \mathrm{~Hz}$, and $55.05 \mathrm{~Hz}$, and the corresponding vibration modes are shown in Figure 9. During the test, the beam body adopts single-piece steel beam and double-piece steel beam to simulate the increase of bridge mass. The first three tested vertical frequencies of the single-piece steel beam are $6.39 \mathrm{~Hz}, 25.45 \mathrm{~Hz}$, and $54.33 \mathrm{~Hz}$. The first three vertical frequencies of the double-piece steel beam are $6.29 \mathrm{~Hz}, 25.17 \mathrm{~Hz}$, and $55.32 \mathrm{~Hz}$. The time history curve and frequency spectrum of the test are shown in Figures 10 and 11 and the structural parameters of the single-piece steel beam are shown in Table 3. 


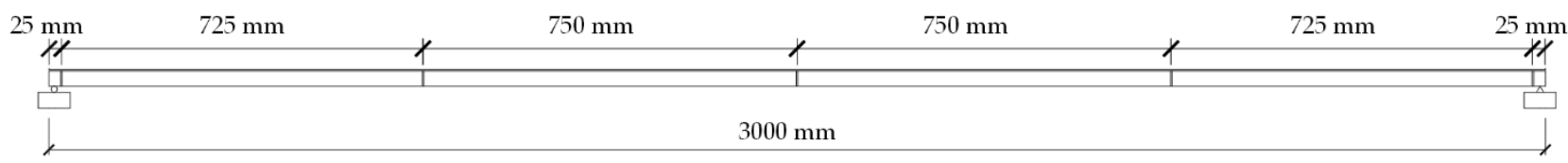

(a)

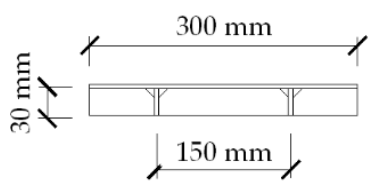

(b)

Figure 8. Design drawing of the steel beam: (a) main view and (b) side view.

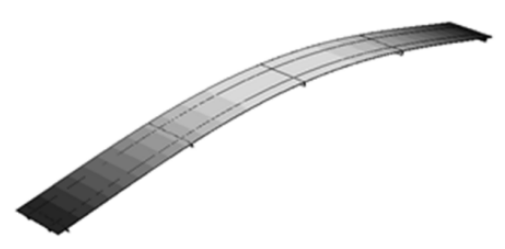

(a)

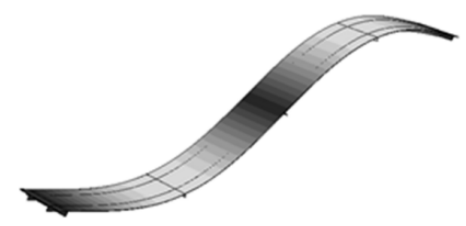

(b)

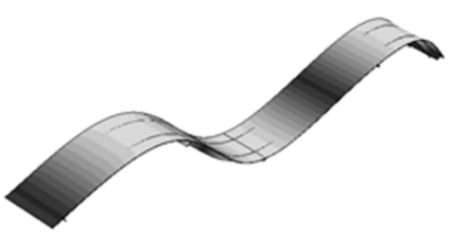

(c)

Figure 9. The first three vertical modes of the steel beam: (a) $7.00 \mathrm{~Hz}$, (b) $25.24 \mathrm{~Hz}$, and (c) $55.05 \mathrm{~Hz}$.

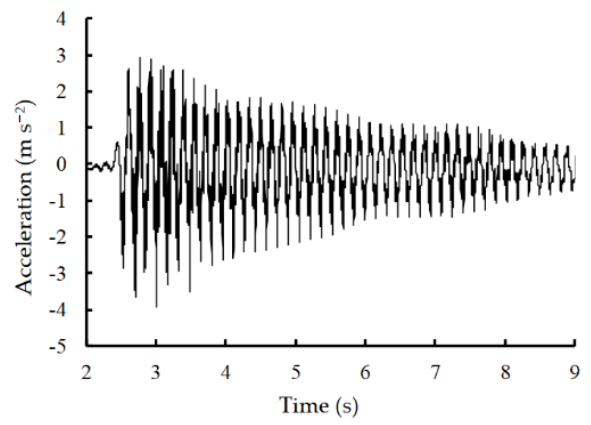

(a)

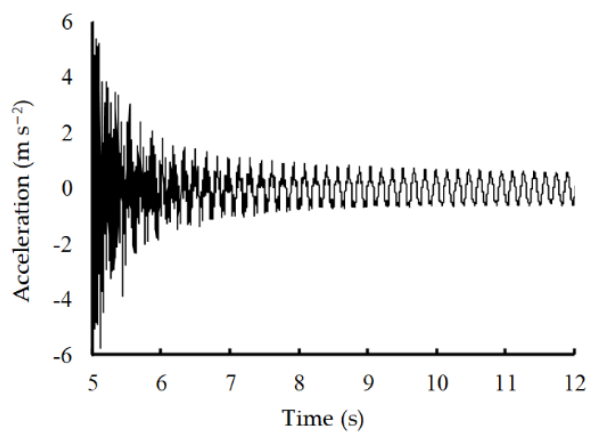

(b)

Figure 10. Vertical acceleration response of free vibration at $1 / 4$ span: (a) single-piece and (b) doublepiece steel beam. 


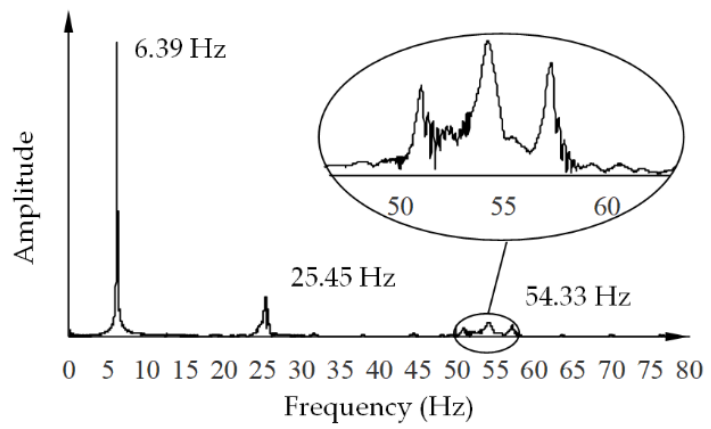

(a)

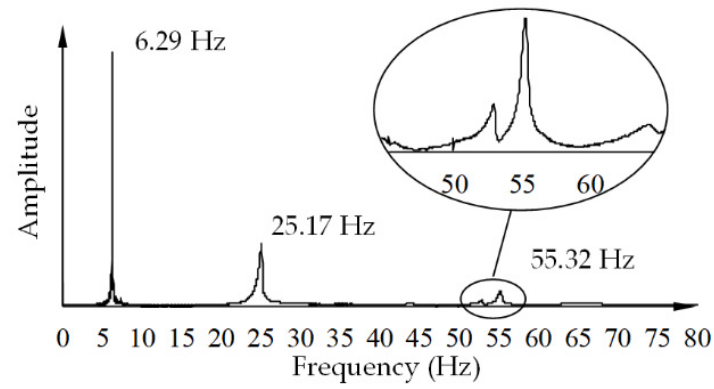

(b)

Figure 11. Vertical acceleration spectrum of free vibration at $1 / 4$ span: (a) single-piece and (b) doublepiece steel beam.

Table 3. Structural parameters of single-piece steel beam.

\begin{tabular}{cccc}
\hline Parameters & $L$ & $m_{b}$ & $E I$ \\
\hline Value & $3 \mathrm{~m}$ & $8.6 \mathrm{~kg} / \mathrm{m}$ & $1.22 \times 10^{4} \mathrm{~N} \cdot \mathrm{m}^{2}$ \\
\hline
\end{tabular}

The main structure of the test vehicle is also made of 3-mm thick steel plates, the length and width are $35 \mathrm{~cm}$ and $25 \mathrm{~cm}$, respectively. As shown in Figure 12, the test vehicle is a double-layer structure, with 4 springs symmetrically arranged between the upper and lower layers. Steel plates with dimensions $35 \mathrm{~cm} \times 25 \mathrm{~cm} \times 3 \mathrm{~mm}$ are used as the counterweight above the springs in the upper layer of the vehicle. The frequency of the vehicle itself can be altered by adjusting the mass of the upper structure, which is the number of steel plates, and the stiffness of the spring. The vehicle mass is differentiated into the light and heavy categories. Light vehicles have no upper structure, and their frequency is much higher than the bridge frequency. Heavy vehicles have an upper layer mass of $6.18 \mathrm{~kg}$. The vehicle frequency is tested to be $9.33 \mathrm{~Hz}$. The time history curve and frequency spectrum of the test are shown in Figure 13. In the case of light and heavy vehicles, the vehicle passes through single- and double-steel beams at different speeds, respectively. Specific conditions of the four testing scenarios are listed in Table 4. As shown in Figure 12, the test vehicle is symmetrical. Due to the limitation of the vertical rod, the upper layer of the vehicle is mainly vertical movement, and there is almost no front and rear rocking movement. When the test vehicle is simplified to a one degree-of-freedom oscillator, the front and rear springs of the test vehicle are simplified to a single spring element in parallel. Therefore, when applying Equations (5) and (6), the position of the center of the test vehicle, which is the same distance as the front and rear wheels, is used as the contact point between the vehicle and bridge. 

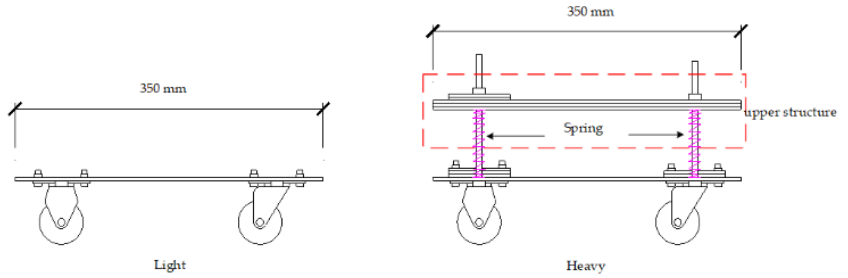

(a)

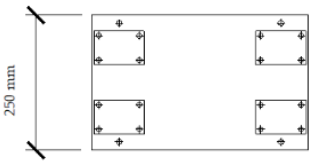

(b)

Figure 12. Design drawing of the test vehicle: (a) main view and (b) top view.

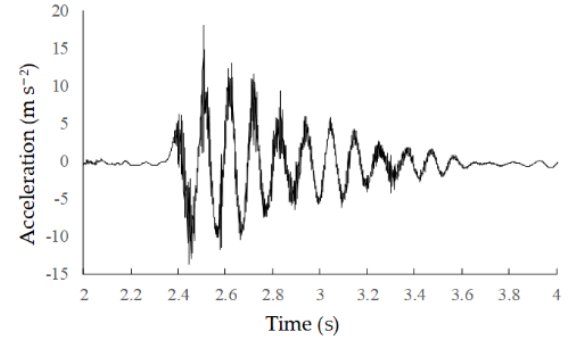

(a)

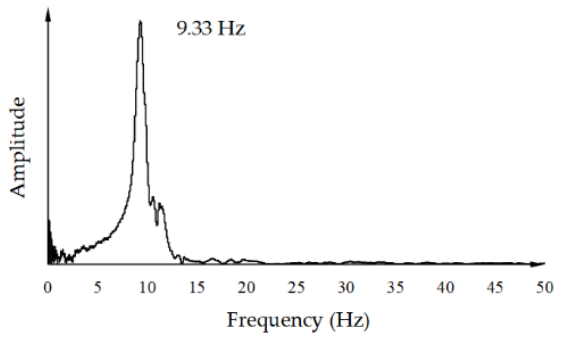

(b)

Figure 13. Free vibration of heavy vehicle: vertical acceleration (a) response and (b) spectrum.

Table 4. Test parameters and results.

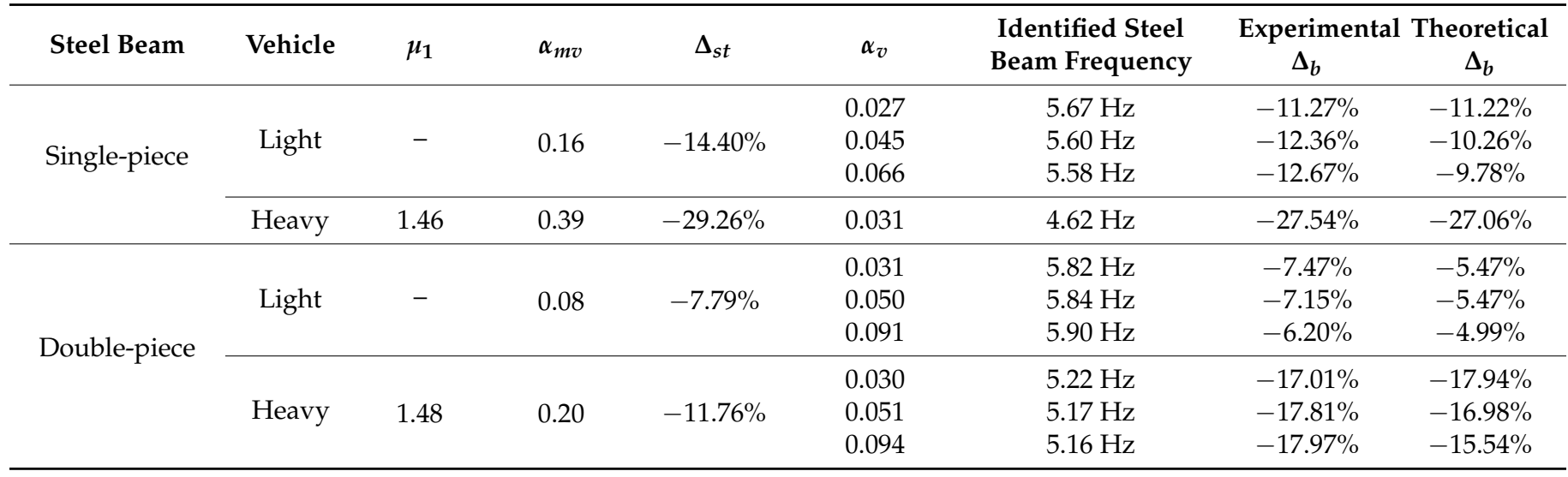

The layout of laboratory experiment is shown in Figure 14. The acceleration sensors are respectively arranged in the center of the vehicle, $1 / 4(3 / 4)$ span and mid span of the bridge, respectively. The speed of the vehicle is controlled by an adjustable speed motor. A one-car length of space, which is $35 \mathrm{~cm}$ away from the two ends, is set as the entry and exit region. Result data excluding the entry and exit region is used for further analysis. At a distance of $35 \mathrm{~cm}$ from the support, a protrusion is set. When the vehicle passes through the protrusion, the acceleration signal will produce a significant fluctuation, which is used as a sign of the vehicle entering and leaving the bridge. A set of vehicle acceleration signals contains four obvious fluctuations, and the data between $0.5 \mathrm{~s}$ after the second fluctuation and $0.5 \mathrm{~s}$ before the third fluctuation are selected for analysis. This ensures that the front and rear wheels of the vehicle are on the bridge at the same time. Moreover, because the speed of the test vehicle passing through the bridge is different, the red box in Figure 15 is only for illustration. The test parameters and results are dimensionless as shown in Table 4. Partial experimental results and their spectrum are shown in Figure 15. To compare the frequency change pattern and provide references for frequency identification, let $\Delta_{s t}$ denote the frequency deviation rate when the vehicle is stationary in the middle of the span. 


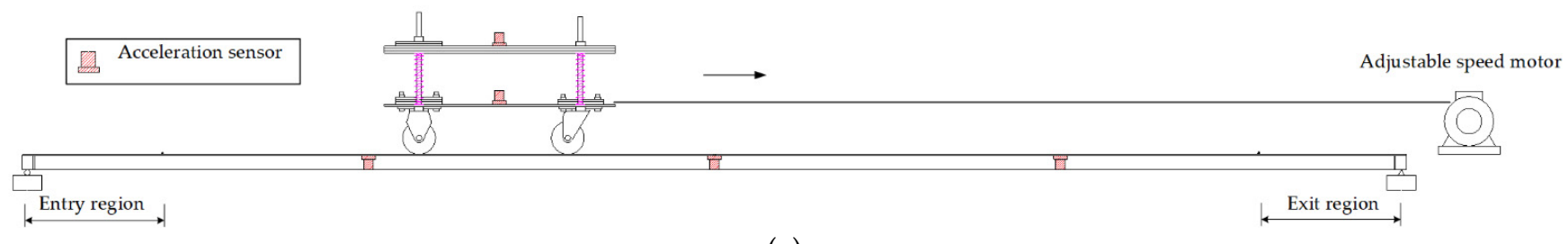

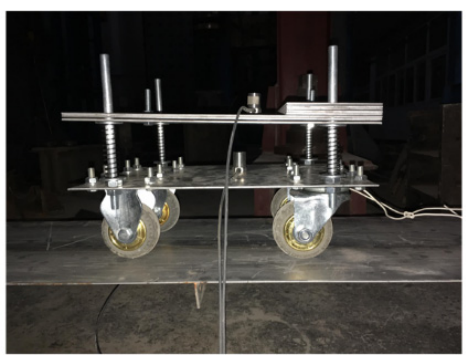

(b) (a)

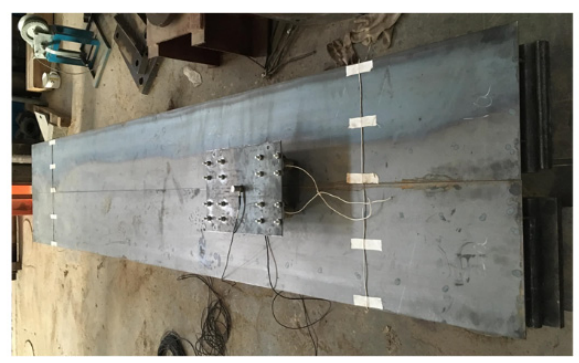

(c)

Figure 14. Layout of experiment: (a) laboratory model; (b) vehicle; and (c) steel beam.

From Table 4, most of the test results show that the measured frequency deviation is larger than the theoretical simulated value. The theoretical $\Delta_{b}$ differs from the experimental $\Delta_{b}$ by about $12 \%$ on average. It is obvious that with the change of $\alpha_{m v}$, the identified steel beam frequency changes significantly. As $\alpha_{m v}$ increases, the degree of the identified bridges frequency deviation increases. If the bridge frequency deviation rate $\left|\Delta_{b}\right|<10 \%$ is used as the condition for successful identification, the steel beam frequency is successfully identified only when the light vehicle and double-piece beam are used. This corresponds to the conditions for identifying success in Section 4.3, that is $\alpha_{m v}<0.1$. In this case, the changing pattern of $\Delta_{b}$ is in good agreement with the theory, that is, $\left|\Delta_{b}\right|$ decreases with the increase of $\alpha_{v}$. After the test vehicle speed is converted into a dimensionless parameter by using the expression in Table A1, the value of $\alpha_{v}$ is only 0.027 0.094 due to the limitation of the experimental conditions. However, in the parameter analysis in Section 4, the value of $\alpha_{v}$ ranges from 0.03 to 0.75 . As a result, in the test results, the effect of $\alpha_{v}$ on $\left|\Delta_{b}\right|$ cannot be well reflected. In this study, the magnitudes of these frequency deviation rates are also greater than those frequency deviation rates calculated by Equation (11). Although, experimental results are limited because of the chosen scaling of the experimental models, the bridge frequency deviation obtained by the bridge dynamic response should be taken seriously. In particular, the bridge frequency deviation caused by passing vehicles must be excluded.

The overall test results are in good agreement with the theoretical calculations and mostly conform to the pattern described in the previous section. However, there are inevitable deviations between the test results and theoretical calculations. For the abnormal phenomena in the above test results, possible reasons for preliminary analysis are as follows: 1. One degree-of-freedom oscillator is used as a vehicle model in the theoretical derivation, which is not the case for an actual vehicle. 2 . The vehicle damping and beam damping are ignored in the theoretical calculation. 3. Improper installation of vehicle components and unsmoothed wheels will affect the interaction between the vehicle and bridge to a certain extent, which, in turn, interferes with the signal. 4. The vehicle will experience a short period of acceleration until reaching the desired speed. Although a previous study [51] shows that little difference exists between the vehicle passing the bridge at a constant speed or constant acceleration. Due to the large vehicle-to-bridge mass ratio in this test, the influence of the non-uniform speed may be amplified, resulting in errors. 


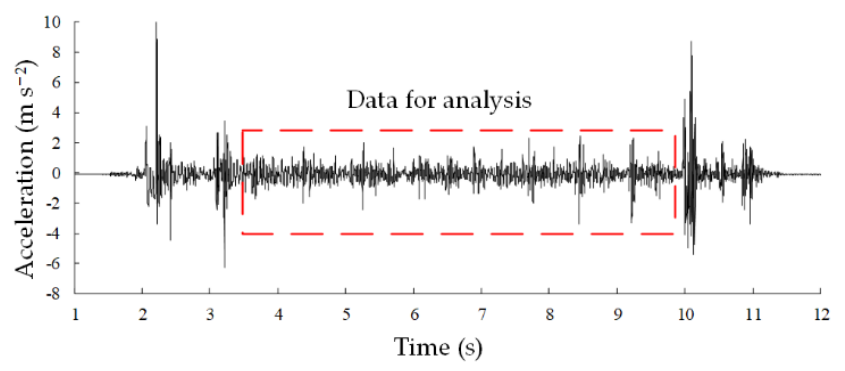

(a)

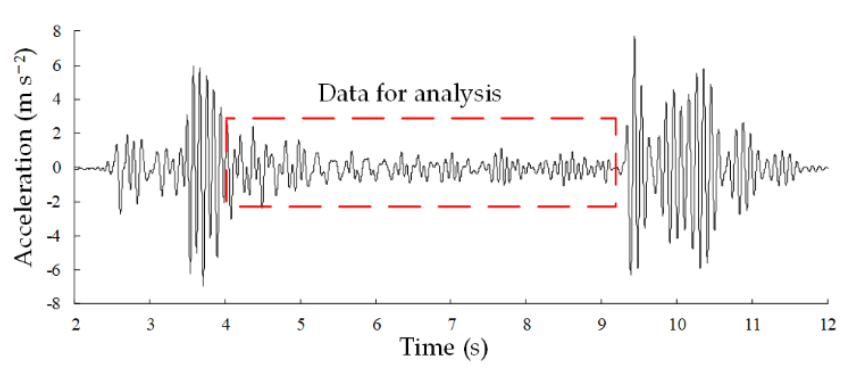

(c)

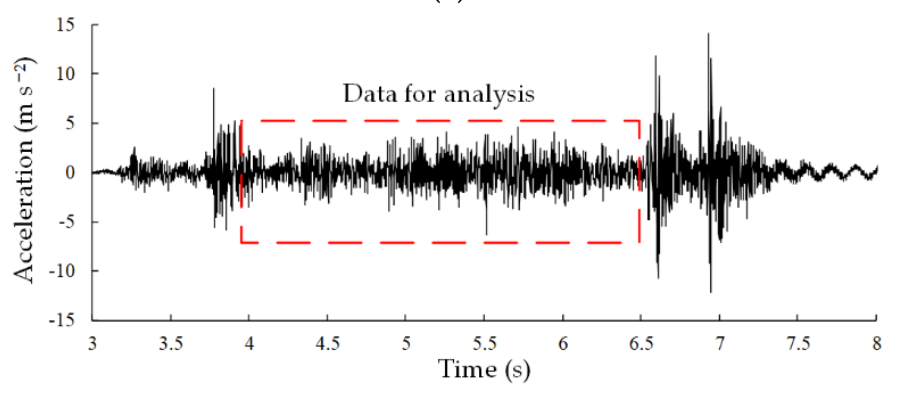

(e)

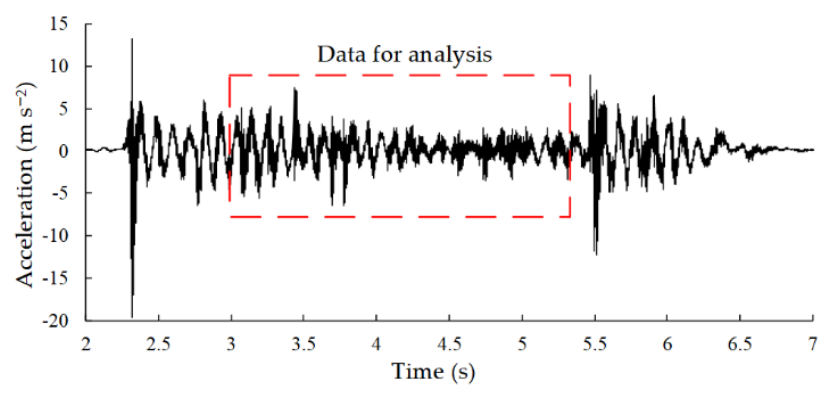

(g)

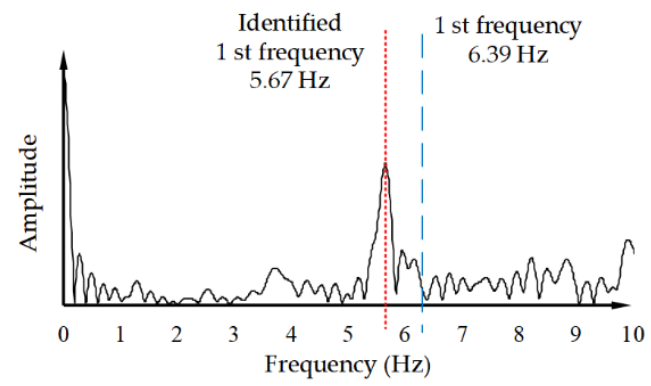

(b)

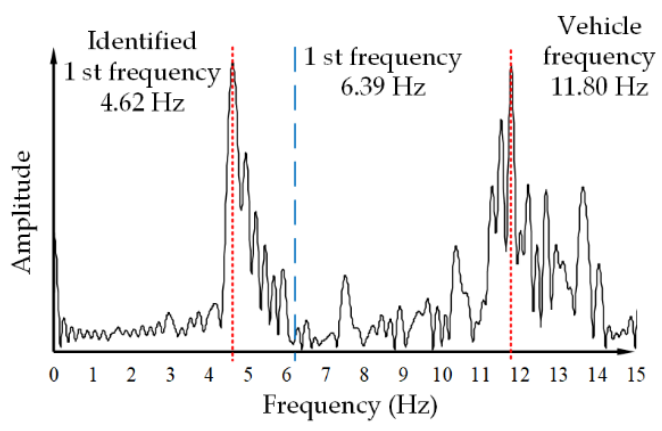

(d)

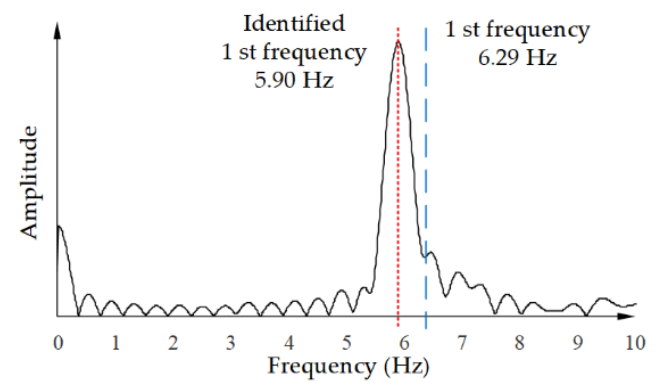

(f)

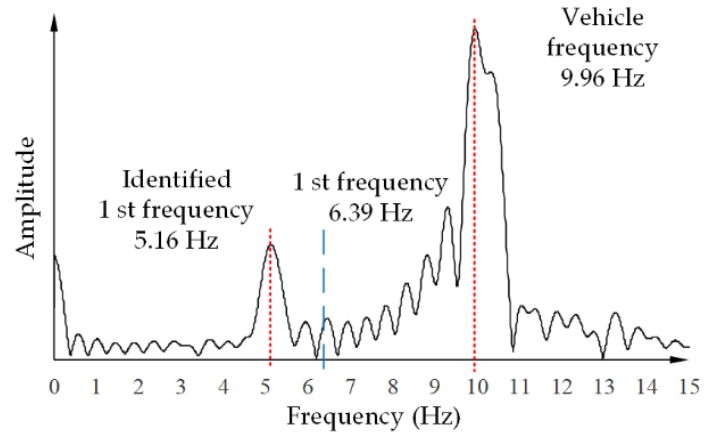

(h)

Figure 15. Vertical acceleration response and spectrum of vehicle: $(\mathbf{a}, \mathbf{b})$ light vehicle passing through single-piece steel beam with $v=0.027 ;(\mathbf{c}, \mathbf{d})$ heavy vehicle passing through single-piece steel beam with $v=0.031 ;(\mathbf{e}, \mathbf{f})$ light vehicle passing through double-piece steel beam with $v=0.065$; and $(\mathbf{g}, \mathbf{h})$ heavy vehicle passing through single-piece steel beam with $v=0.066$.

\section{Conclusions}

In this paper, the one degree-of-freedom oscillator and a simply-supported EulerBernoulli beam model were used to establish a vehicle-bridge interaction system. With a large vehicle-bridge mass ratio, dimensionless parameters, mainly from the three aspects, vehicle speed, vehicle-bridge frequency ratio, and mass ratio, were used to discuss the patterns of an extracted bridge fundamental frequency from the acceleration signal of a 
passing vehicle. In general, under the influence of vehicle mass, the bridge fundamental frequency identified from the vehicle acceleration signal shows a different pattern than that of when the vehicle mass is ignored. An intuitive display of the accuracy of the extracted bridge fundamental frequency is given. Through the model test in the laboratory, the theoretically calculated patterns are verified. In addition, the corresponding screening principles and methods are proposed on how to select the appropriate peak frequency from the spectrogram to achieve successful bridge frequency identification. The main conclusions are summarized as follows:

1. The right-biased frequency does not always occupy the main advantage in the identified bridge fundamental frequencies, especially when $\mu_{1}>1$, the identified bridge fundamental frequency is dominated by the left-biased frequency.

2. When $\mu_{1}>1, \Delta_{b}$ is greatly affected by $\alpha_{m v}$ and $\alpha_{v}$, and the interaction between $\mu_{1}$ and the other two parameters is small; when $\mu_{1}<1, \mu_{1}, \alpha_{m v}$, and $\alpha_{v}$ have a greater impact on $\Delta_{b}$, and there is a similar degree of interaction between them.

3. To ensure the accuracy of identifying the bridge fundamental frequency, the vehiclebridge mass ratio and vehicle speed should not be too large. It is recommended that the vehicle-bridge mass ratio should not exceed $10 \%$, or even $5 \%$.

4. This paper proposed a few basic principles and reference methods for effective bridge frequency identification using spectrograms of vehicle acceleration, which provide greater accuracy and rigorous for bridge frequency extraction.

The conclusions of this paper can provide useful suggestions for the application of bridge indirect monitoring techniques. Due to the limitations of the simplified model and the scale of the experimental model, further investigations are required in order to understand the pattern of bridge frequency deviation. First, the models of vehicles and bridges should be refined. In future parametric analyses, the effects of vehicle damping and bridge damping on identifying bridge frequencies from a passing vehicle's acceleration will be considered. Second, field tests will be carried out. For a real bridge, the conditions under which the frequency deviation phenomenon occurs should be studied.

Author Contributions: Conceptualization, B.H.; methodology, Q.W. and H.X.; software, Q.W. and W.Y.; validation, Q.W., B.H. and J.Y.; formal analysis, Q.W.; investigation, Q.W. and W.Y.; writingoriginal draft preparation, Q.W.; writing-review and editing, B.H., J.Z. and H.X.; visualization, Q.W.; supervision, B.H. and J.Z.; project administration, B.H. All authors have read and agreed to the published version of the manuscript.

Funding: This research was funded by the Fundamental Research Funds for the Central Universities under grant no. 2018YJS102; the Science and Technology Project of Beijing Transportation Commission under grant no. KJ-2017-5; and the Fundamental Research Funds for the Central Universities under grant no. 2019RC012.

Institutional Review Board Statement: Not applicable.

Informed Consent Statement: Not applicable.

Data Availability Statement: Not applicable.

Conflicts of Interest: The authors declare no conflict of interest.

\section{Appendix A}

The expressions for $\alpha_{y b}, \alpha_{y v}, \alpha_{m v}, \alpha_{m w}, \alpha_{v}, s, \tau$, and $g^{*}$ are shown in Table A1. 
Table A1. Structural parameters of single-piece steel beam.

\begin{tabular}{ccc}
\hline Parameters & Expression & Implication \\
\hline$\alpha_{y b}$ & $y_{b} / L$ & bridge vertical displacement \\
$\alpha_{y v}$ & $y_{v} / L$ & vehicle vertical displacement \\
$\alpha_{m v}$ & $m_{v} / m_{b} L$ & vehicle mass \\
$\alpha_{m w}$ & $m_{w} / m_{b} L$ & wheel mass \\
$\alpha_{v}$ & $\sqrt{\frac{m_{b}}{E I}} v L$ & vehicle speed \\
$s$ & $x / L$ & vehicle position \\
$\tau$ & $\sqrt{\frac{E I}{m_{b}}} t / L^{2}$ & time \\
$g^{*}$ & $\frac{m_{b} L^{3}}{E I} g$ & acceleration due to gravity \\
\hline
\end{tabular}

\section{Appendix B}

Substituting Equation (8) into Equation (6), vehicle response $\alpha_{y v}$ can be solved from Equation (6) by Duhamel's integral. Then, $\alpha_{y v}$ can be differentiated twice to yield the acceleration of the vehicle as:

$$
\begin{array}{r}
\ddot{\alpha}_{y v}=\sum_{n=1}^{\infty} \frac{2\left(\alpha_{m v}+\alpha_{m w}\right) g^{*} \Omega_{v}^{2}}{\Omega_{b n}^{2}\left(1-S_{n}^{2}\right)}\left[\cos \Omega_{v} \tau+\frac{\left(2 S_{n} / \mu_{n}\right)^{2} \cos 2 n \pi s-\cos \Omega_{v} \tau}{1-\left(2 S_{n} / \mu_{n}\right)^{2}}\right. \\
+S_{n} \frac{\left(1-S_{n}\right)^{2} / \mu_{n}^{2} \cos \left(\Omega_{b n}-n \pi \alpha_{v}\right) \tau-\cos \Omega_{v} \tau}{1-\left(1-S_{n}\right)^{2} / \mu_{n}^{2}} \\
\left.-S_{n} \frac{\left(1+S_{n}\right)^{2} / \mu_{n}^{2} \cos \left(\Omega_{b n}+n \pi v_{v}\right) \tau-\cos \Omega_{v} \tau}{1-\left(1+S_{n}\right)^{2} / \mu_{n}^{2}}\right] .
\end{array}
$$

After simplification, the expressions of $A 1, A 2, A 3$, and $A 4$ are as follows:

$$
\begin{gathered}
A_{1}=\sum_{n=1}^{\infty} \frac{2\left(\alpha_{m v}+\alpha_{m w}\right) g^{*} \Omega_{v}^{2}}{\Omega_{b n}^{2}\left(1-S_{n}^{2}\right)}\left[-\frac{\left(2 S_{n} / \mu_{n}\right)^{2}}{1-\left(2 S_{n} / \mu_{n}\right)^{2}}-\frac{S_{n}}{1-\left(1-S_{n}\right)^{2} / \mu_{n}^{2}}+\frac{S_{n}}{1-\left(1+S_{n}\right)^{2} / \mu_{n}^{2}}\right] \\
A_{2}=\sum_{n=1}^{\infty} \frac{2\left(\alpha_{m v}+\alpha_{m w}\right) g^{*} \Omega_{v}^{2}}{\Omega_{b n}^{2}\left(1-S_{n}^{2}\right)}\left[\frac{\left(2 S_{n} / \mu_{n}\right)^{2}}{1-\left(2 S_{n} / \mu_{n}\right)^{2}}\right] \\
A_{3}=\sum_{n=1}^{\infty} \frac{2\left(\alpha_{m v}+\alpha_{m w}\right) g^{*} \Omega_{v}^{2}}{\Omega_{b n}^{2}\left(1-S_{n}^{2}\right)}\left[\frac{S_{n}\left(1-S_{n}\right)^{2}}{\mu_{n}^{2}-\left(1-S_{n}\right)^{2}}\right] \\
A_{4}=\sum_{n=1}^{\infty} \frac{2\left(\alpha_{m v}+\alpha_{m w}\right) g^{*} \Omega_{v}^{2}}{\Omega_{b n}^{2}\left(1-S_{n}^{2}\right)}\left[-\frac{S_{n}\left(1+S_{n}\right)^{2}}{\mu_{n}^{2}-\left(1+S_{n}\right)^{2}}\right] .
\end{gathered}
$$

\section{References}

1. Wu, B.; Wu, G.; Yang, C. Parametric Study of a Rapid Bridge Assessment Method Using Distributed Macro-Strain Influence Envelope Line. Mech. Syst. Signal Processing 2019, 120, 642-663. [CrossRef]

2. Fan, W.; Qiao, P. Vibration-Based Damage Identification Methods: A Review and Comparative Study. Struct. Health Monit. 2010, 10, 83-111. [CrossRef]

3. Zhu, X.; Law, S. Recent Developments in Inverse Problems of Vehicle-Bridge Interaction Dynamics. J. Civ. Struct. Health Monit. 2016, 6, 107-128. [CrossRef]

4. Yang, Y.B.; Wang, Z.-L.; Shi, K.; Xu, H.; Wu, Y.T. State-of-the-Art of Vehicle-Based Methods for Detecting Various Properties of Highway Bridges and Railway Tracks. Int. J. Struct. Stab. Dyn. 2020, 20. [CrossRef]

5. Yang, Y.; Lin, C.; Yau, J. Extracting Bridge Frequencies From The Dynamic Response Of A Passing Vehicle. J. Sound Vib. 2004, 272, 471-493. [CrossRef]

6. Cunha, A.; Caetano, E.; Delgado, R. Dynamic Tests on Large Cable-Stayed Bridge. J. Bridge Eng. 2001, 6, 54-62. [CrossRef]

7. Conte, J.; He, X.; Moaveni, B.; Masri, S.; Caffrey, J.; Wahbeh, M.; Tasbihgoo, F.; Whang, D.; Elgamal, A. Dynamic Testing of Alfred Zampa Memorial Bridge. J. Struct. Eng. 2008, 134, 1006-1015. [CrossRef]

8. Brownjohn, J; Moyo, P.; Omenzetter, P.; Lu, Y. Assessment of Highway Bridge Upgrading by Dynamic Testing and Finite-Element Model Updating. J. Bridge Eng. 2003, 8, 162-172. [CrossRef]

9. Yang, Y.; Chang, K. Extracting the Bridge Frequencies Indirectly from a Passing Vehicle: Parametric Study. Eng. Struct. 2009, 31, 2448-2459. [CrossRef] 
10. Kong, X.; Cai, C.; Deng, L.; Zhang, W. Using Dynamic Responses of Moving Vehicles to Extract Bridge Modal Properties of a Field Bridge. J. Bridge Eng. 2017, 22, 04017018. [CrossRef]

11. Wang, Z.; Chen, S.; Lederman, G.; Cerda, F.; Bielak, J.; Garrett, J.H.; Rizzo, P.; Kovačević, J. Comparison of Sparse Representation and Fourier Discriminant Methods: Damage Location Classification in Indirect Lab-Scale Bridge Structural Health Monitoring. In Structures Congress; American Society of Civil Engineers: Pittsburgh, PA, USA, 2013; pp. 436-446.

12. Kim, C.; Chang, K.; Mcgetrick, P.; Inoue, S.; Hasegawa, S. Utilizing Moving Vehicles as Sensors for Bridge Condition ScreeningA Laboratory Verification. Sens. Mater. 2017, 29, 153.

13. McGetrick, P.; Kim, C.; González, A.; Brien, E. Experimental Validation of a Drive-By Stiffness Identification Method for Bridge Monitoring. Struct. Health Monit. 2015, 14, 317-331. [CrossRef]

14. Lin, C.; Yang, Y. Use of a Passing Vehicle to Scan the Fundamental Bridge Frequencies: An Experimental Verification. Eng. Struct. 2005, 27, 1865-1878. [CrossRef]

15. Nagayama, T.; Reksowardojo, A.; Su, D.; Mizutani, T. Bridge Natural Frequency Estimation by Extracting the Common Vibration Component from the Responses of Two Vehicles. Eng. Struct. 2017, 150, 821-829. [CrossRef]

16. Lederman, G.; Chen, S.; Garrett, J.; Kovačević, J.; Noh, H.; Bielak, J. Track Monitoring from the Dynamic Response of a Passing Train: A Sparse Approach. Mech. Syst. Signal Processing 2017, 90, 141-153. [CrossRef]

17. Malekjafarian, A.; O'Brien, E. On the Use of a Passing Vehicle for the Estimation of Bridge Mode Shapes. J. Sound Vib. 2017, 397, 77-91. [CrossRef]

18. Yang, Y.; Zhang, B.; Chen, Y.; Qian, Y.; Wu, Y. Bridge Damping Identification by Vehicle Scanning Method. Eng. Struct. 2019, 183, 637-645. [CrossRef]

19. Malekjafarian, A.; O'Brien, E. Identification of Bridge Mode Shapes Using Short Time Frequency Domain Decomposition of the Responses Measured in a Passing Vehicle. Eng. Struct. 2014, 81, 386-397. [CrossRef]

20. Yang, Y.B.; Zhang, B.; Qian, Y.; Wu, Y.T. Contact-Point Response for Modal Identification of Bridges by a Moving Test Vehicle. Int. J. Struct. Stab. Dyn. 2018, 18, 5. [CrossRef]

21. Yang, Y.B.; Shi, K.; Wang, Z.L.; Xu, H.; Zhang, B.; Wu, Y.T. Using a Single-DOF Test Vehicle to Simultaneously Retrieve the First Few Frequencies and Damping Ratios of the Bridge. Int. J. Struct. Stab. Dyn. 2021, 21, 2150108. [CrossRef]

22. Xu, H.; Huang, C.C.; Wang, Z.L.; Shi, K.; Wu, Y.T.; Yang, Y.B. Damped test vehicle for scanning bridge frequencies: Theory, simulation and experiment. J. Sound Vib. 2021, 506, 116155. [CrossRef]

23. Jin, N.; Yang, Y.B.; Dimitrakopoulos, E.G.; Paraskeva, T.S.; Katafygiotis, L.S. Application of short-time stochastic subspace identification to estimate bridge frequencies from a traversing vehicle. Eng. Struct. 2021, 230, 111688. [CrossRef]

24. Yang, Y.B.; Xu, H.; Mo, X.Q.; Wang, Z.L.; Wu, Y.T. An effective procedure for extracting the first few bridge frequencies from a test vehicle. Acta Mech. 2021, 232, 1227-1251. [CrossRef]

25. Yang, Y.B.; Xu, H.; Wang, Z.L.; Shi, K.; Wu, Y.T. Refined detection technique for bridge frequencies using rocking motion of single-axle moving vehicle. Mech. Syst. Signal Processing 2022, 162, 107992. [CrossRef]

26. Obrien, E.J.; Malekjafarian, A. A mode shape-based damage detection approach using laser measurement from a vehicle crossing a simply supported bridge. Struct. Control Health Monit. 2016, 23, 1273-1286. [CrossRef]

27. Harris, N.; Gonzalez, A.; OBrien, E.; McGetrick, P. Characterisation of Pavement Profile Heights Using Accelerometer Readings and a Combinatorial Optimisation Technique. J. Sound Vib. 2010, 329, 497-508. [CrossRef]

28. Yang, Y.; Li, Y.; Chang, K. Using Two Connected Vehicles to Measure the Frequencies of Bridges with Rough Surface: A Theoretical Study. Acta Mech. 2012, 223, 1851-1861. [CrossRef]

29. Yang, Y.; Chang, K.; Li, Y. Filtering Techniques for Extracting Bridge Frequencies from a Test Vehicle Moving over the Bridge. Eng. Struct. 2013, 48, 353-362. [CrossRef]

30. Wang, H.; Nagayama, T.; Nakasuka, J.; Zhao, B.; Su, D. Extraction of Bridge Fundamental Frequency from Estimated Vehicle Excitation through a Particle Filter Approach. J. Sound Vib. 2018, 428, 44-58. [CrossRef]

31. Hester, D.; González, A. A Discussion on the Merits And Limitations of Using Drive-by Monitoring to Detect Localised Damage In A Bridge. Mech. Syst. Signal Processing 2017, 90, 234-253. [CrossRef]

32. Siringoringo, D.; Fujino, Y. Estimating Bridge Fundamental Frequency From Vibration Response of Instrumented Passing Vehicle: Analytical and Experimental Study. Adv. Struct. Eng. 2012, 15, 417-433. [CrossRef]

33. Yang, Y.; Lin, C. Vehicle-Bridge Interaction Dynamics And Potential Applications. J. Sound Vib. 2005, 284, 205-226. [CrossRef]

34. Oshima, Y.; Yamamoto, K.; Sugiura, K. Damage Assessment of a Bridge Based on Mode Shapes Estimated by Responses of Passing Vehicles. Smart Struct. Syst. 2014, 13, 731-753. [CrossRef]

35. Yang, Y.; Li, Y.; Chang, K. Constructing the Mode Shapes of a Bridge from a Passing Vehicle: A Theoretical Study. Smart Struct. Syst. 2014, 13, 797-819. [CrossRef]

36. Nguyen, K.; Tran, H. Multi-Cracks Detection of a Beam-Like Structure Based on the On-Vehicle Vibration Signal and Wavelet Analysis. J. Sound Vib. 2010, 329, 4455-4465. [CrossRef]

37. Chen, S.; Cerda, F.; Rizzo, P.; Bielak, J.; Garrett, J.; Kovacevic, J. Semi-Supervised Multiresolution Classification Using Adaptive Graph Filtering with Application to Indirect Bridge Structural Health Monitoring. IEEE Trans. Signal Processing 2014, 62, 2879-2893. [CrossRef]

38. Cerda, F.; Chen, S.; Bielak, J.; Garrett, J.; Rizzo, P.; Kovacevic, J. Indirect Structural Health Monitoring of a Simplified LaboratoryScale Bridge Model. Smart Struct. Syst. 2014, 13, 849-868. [CrossRef] 
39. Shi, Z.; Uddin, N. Extracting Multiple Bridge Frequencies from Test Vehicle-A Theoretical Study. J. Sound Vib. 2021, 490, 115735. [CrossRef]

40. Kalil, E.; Debarshi, S.; Satish, N.; Sun, L. Vibration-based structural health monitoring under changing environmental conditions using Kalman filtering. Mech. Syst. Signal Processing 2019, 117, 1-15.

41. Anastasopoulos, D.; Roeck, G.D.; Reynders, E. One-year operational modal analysis of a steel bridge from high-resolution macrostrain monitoring: Influence of temperature vs. retrofitting. Mech. Syst. Signal Processing 2021, 161, 107951. [CrossRef]

42. Chang, K.C.; Kim, C.W.; Borjigin, S. Variability in bridge frequency induced by a parked vehicle. Smart Struct. Syst. 2014, 13, 755-773. [CrossRef]

43. Zhang, Q.W.; Fan, L.C.; Yuan, W.C. Traffic-induced variability in dynamic properties of cable-stayed bridge. Earthq. Eng. Struct. Dyn. 2010, 31, 2015-2021. [CrossRef]

44. Górski, P.; Napieraj, M.; Konopka, E. Variability evaluation of dynamic characteristics of highway steel bridge based on daily traffic-induced vibrations. Measurement 2020, 164, 108074. [CrossRef]

45. Kim, C.; Jung, D.S.; Kim, N.S.; Yoon, J.G. Effect of Vehicle Mass on the Measured Dynamic Characteristics of Bridges from TrafficInduced Vibration Test. In Proceedings of the 19th International Modal Analysis Conference, Orlando, FL, USA, 5-8 February 2001; pp. 1106-1111.

46. Feng, S.D.M. Effect of vehicle weight on natural frequencies of bridges measured from traffic-induced vibration. Earthq. Eng. Struct. Dyn. 2003, 2, 109-116.

47. Cantero, D.; McGetrick, P.; Kim, C.; O’Brien, E. Experimental Monitoring of Bridge Frequency Evolution during the Passage Of Vehicles with Different Suspension Properties. Eng. Struct. 2019, 187, 209-219. [CrossRef]

48. Yang, Y.; Cheng, M.; Chang, K. Frequency Variation in Vehicle-Bridge Interaction Systems. Int. J. Struct. Stab. Dyn. 2013, 13, 1350019. [CrossRef]

49. Yu, F.; Li, Y. Vehicle System Dynamics; China Machine Press: Beijing, China, 2005.

50. Morris, M. Factorial Sampling Plans For Preliminary Computational Experiments. Technometrics 1991, 33, 161-174. [CrossRef]

51. Xia, H.; Zhang, N.; Guo, W. Coupling Vibrations of Train-Bridge System; Science Press: Beijing, China, 2014. 